\title{
Das Gesprächsführungspraktikum im 2. Studienjahr des Modellstudiengangs HannibaL: Eine Evaluation mittels Selbsteinschätzungen der Studierenden
}

\section{Zusammenfassung}

Im MHH-Modellstudiengang HannibaL werden Gesprächsführungskompetenzen für Anamneseerhebung und Diagnosemitteilung durch eine Objective Structured Clinical Examination (OSCE) geprüft, die Teil der dem Ersten Abschnitt der Ärztlichen Prüfung äquivalenten Prüfungen sind. Das vorbereitende Gesprächsführungspraktikum im 2. Studienjahr wurde 2009/10 evaluiert.

Mittels lernzielspezifischer Fragebogen wurden der Lernbedarf erhoben, Vorher-Nachher-Vergleiche der selbsteingeschätzten Kompetenzen durchgeführt und zentrale Lehrmethoden bewertet (5-Punkt-Likertskalen, „5“=hohe Ausprägung). Zu TO (Beginn des Praktikums) nahmen 267 Studierende teil (Teilnahmerate: 93,7\%), von denen 180 den T1-Fragebogen beim letzten Praktikumstermin ausfüllten (67,4\%). Es wurden Varianzanalysen mit Messwiederholungsfaktor und T-Tests für verbundene Stichproben durchgeführt.

Die höchsten Lernbedarfe zeigten sich bei den „to show how“-Items zu Anamneseerhebung und Diagnosemitteilung $(M=4,4)$. Die T1-TO-Vergleiche zeigten die größten Verbesserungen bei den anamnesespezifischen Items (,to know how“: Mittelwertsdifferenz=+1,7, „to show how“:+1,8, $p<.0001$ wie bei allen Tests) und beim „to know how“-Item zur Diagnosemitteilung $(+1,6)$, gefolgt von der Umsetzung einer Diagnosemitteilung $(+1,4)$, partizipativer Entscheidungsfindung $(+1,2)$, der Einschätzung eigener Stärken/Schwächen $(+1,0)$ und dem sicheren Zugehen auf neue Patienten $(+0,7)$. Studierende mit TO-Werten von „1“ oder „2“ auf den jeweiligen Skalen verbesserten sich über alle Items im Mittel um 2,2 Punkte, solche mit „3“ um 1,1, und mit „4“ oder „5“ um 0,1. Methodisch wurde der Einsatz der Simulationspatienten am hilfreichsten bewertet ( $M=4,8 ; 87 \%$ mit dem Wert „5“).

Das Gesprächsführungspraktikum ist bezüglich aller zentralen Lernziele mit deutlichen Lernfortschritten assoziiert. Methodisch wird vor allem der Einsatz von Simulationspatienten (pro Praktikumsgruppe mit 10 Studierenden zu 3 von 7 Terminen mit jeweils 2-4 Simulationspatienten) am besten bewertet. Die Evaluation spricht für einen weiteren Ausbau des Gesprächsführungscurriculums an der $\mathrm{MHH}$ und von hochschul-/ fakultätsübergreifenden Aktivitäten, die abschließend diskutiert werden.

\author{
Thomas von Lengerke ${ }^{1}$ \\ Angelika Kursch ${ }^{1}$ \\ Karin Lange ${ }^{1}$
}

\$3* $\square$ HUWam MHH* ${ }^{1}$

1 Medizinische Hochschule Hannover, Forschungs- und Lehreinheit Medizinische Psychologie, Hannover, Deutschland

Schlüsselwörter: Arzt-Patient-Gesprächsführung, Ausbildung im

Medizinstudium, Anamneseerhebung, Diagnosemitteilung,

Gesundheitskommunikation

\section{Einleitung}

Durch die Neufassung der Approbationsordnung für Ärzte [1], die in $\$ 1$ als ein neues Ziel der Ausbildung die Vermittlung praktischer Erfahrungen im Umgang mit Patienten spezifiziert, sowie den Nationalen Kompetenzbasierten Lernzielkatalog Medizin (NKLM) [2] hat die Vermittlung sozial-kommunikativer Kompetenzen im Medizinstudium eine zentrale curriculare Bedeutung. Auch der Modellstudiengang HannibaL (Hannoverscher integrierter, berufsorientierter und adaptiver Lehrplan) der Medizinischen Hochschule Hannover ( $\mathrm{MHH}$ ) stellt den praktischen Umgang mit Patienten und ihren Erkrankungen in den Mittelpunkt. In ihm werden seit dem WS 2005/06 alle neu an der $\mathrm{MHH}$ immatrikulierten Studierenden ausgebildet.

Zu HannibaL gehört auch ein 28-stündiges Praktikum zur Arzt-Patient-Gesprächsführung. Es wird von den Forschungs- und Lehreinheiten Medizinische Psychologie und Medizinische Soziologie in Kooperation mit dem In- 
stitut für Allgemeinmedizin, der Klinik für Psychosomatik und Psychotherapie sowie des Arbeitsbereichs Klinische Psychologie der Klinik für Psychiatrie, Sozialpsychiatrie und Psychotherapie angeboten. Seine Lernziele bestehen aus grundlegenden Kompetenzen in der Erhebung von Anamnesen, der Vermittlung von Diagnosen sowie der Anwendung partizipativer Entscheidungsfindung (PEF) in diesem Rahmen. Diese Kompetenzen werden an zwei von sechs Stationen einer Objective Structured Clinical Examination (OSCE) geprüft. Diese ist ein Teil der hochschulinternen Prüfungen, die in HannibaL den Ersten Abschnitt der Ärztlichen Prüfung ersetzen. An den anderen vier OSCE-Stationen werden Untersuchungstechniken geprüft, die in den anderen Teilen des Moduls „Diagnostische Methoden“** gelehrt werden, zu dem das Praktikum gehört. Zugleich wurden 2010 im dritten Jahr Simulationspatienten (SP) nicht nur in der OSCE, sondern auch im Praktikum eingesetzt. Über die zentrale Evaluation des Gesamtmoduls hinaus war es daher von Bedeutung, das Praktikum einer im Hinblick auf seine Lernziele spezifischen Evaluation zu unterziehen.

\section{Praktikumskonzept und -inhalte}

Das Praktikum umfasst sieben Termine zu je vier Unterrichtsstunden. Die Studierenden jedes Jahrganges werden in 28 Zehnergruppen eingeteilt, um mehrmaliges Probehandeln in Rollenspielen untereinander und mit SP zu ermöglichen. Der Einsatz von SP ist seit ihrer erstmaligen Beschreibung [3] international zu einem Standard des praktischen Erlernens kommunikativer Kompetenzen im Medizinstudium geworden [4], [5], und inzwischen auch in Deutschland verbreitet [5], [6], [7]. Dabei gilt das Feedback der SP als wichtiger Teil des Lernprozesses [4], [8]. Die hier eingesetzten SP wurden vor allem aus Mitgliedern des Deutschen Diabetiker Bundes (Bezirk Hannover) rekrutiert, und daneben aus Herzgruppen und instruierten Patienten der Abteilung Allgemeinmedizin. In geringerer Zahl werden auch Personen aus einem größeren Pool von Amateurschauspielern eingesetzt, der seit 2007 durch Vermittlung des Amateurtheaterverbandes Niedersachsen aufgebaut werden konnte (in der OSCE machen Amateurschauspieler das Gros der SP aus, wobei jeder SP nur im Praktikum oder der OSCE eingesetzt wird). Insgesamt stehen z. Z. circa 80 SP zwischen 23 und 77 Jahren zur Verfügung (61,3\% Frauen). Ihr Einsatz wird durch angemessene Honorare vergütet.

Tabelle 1 gibt einen Überblick über die sieben Praktikumstermine. In den ersten beiden Einheiten werden die Grundlagen der ärztlichen Gesprächsführung vor dem theoretischen Hintergrund der Axiome von Watzlawick [9] und den Modellen von Schulz von Thun [10] vermittelt. Dabei werden praktische Übungen (z. B. Rollenspiele) vor allem zu aktivem Zuhören, nonverbaler Kommunikation und Gesprächsanfängen eingesetzt.

Vorbereitend zur dritten Einheit erhalten die Studierenden als Selbststudiumsanteil den Auftrag, mit einer älteren Person eine biopsychosoziale Anamneseerhebung zu er- proben. Die Auswertung dieser Erfahrung erfolgt im Plenum zu Beginn der dritten Einheit, die schwerpunktmäßig die inhaltliche und praktische Auseinandersetzung mit der Strukturierung und Durchführung von Anamnesen beinhaltet. Hierbei wird ein Erstkontakt in einer Arztpraxis als Setting zugrunde gelegt. Die Studierenden nutzen zur Vorbereitung und Auswertung der Rollenspiele einen vom APG-Lehrteam entwickelten Beobachtungsbogen, der die wesentlichen Kriterien der Calgary-Cambridge Guides [11], [4], [12] und von FüeßI und Middeke [13] aufgreift. Zur vierten Einheit werden pro Zehnergruppe zwei SP eingeladen, wobei durch ihre Rotation und durch Aufteilen der Gruppen bis zu acht Gespräche pro Praktikumsgruppe geführt und ausgewertet werden können. Zum Trainieren einer biopsychosozialen Anamneseerhebung berichten die SP dabei über eigene Krankheitssymptome (unter Ausschluss aktuell vorhandener Symptome und Notfallsituationen) oder greifen auf vom Dozententeam vorgegebene Fallgeschichten zurück.

Schwerpunktthema der fünften Sitzung ist das verständliche und patientenorientierte Mitteilen einer Diagnose in Anlehnung an die Calgary-Cambridge Guides [11], [4], [12] und SPIKES [14]. Im Rollenspiel üben die Studierenden mit Hilfe eines wiederum spezifischen Beobachtungsbogen das „breaking bad news“ als ärztliche Aufgabe. In der sechsten Sitzung ermöglichen wiederum SP das Ausprobieren des Gelernten. Den Rollenspielen liegen Fallvignetten zugrunde, deren Inhalte den jeweiligen SP angepasst werden. Sie beziehen sich entweder auf Diabetes mellitus Typ 2, Hypertonie, koronare Herzkrankheit, Bandscheibenvorfall, chronische Bronchitis/COPD, gutartige Prostatahyperplasie oder Brustkrebs. Diese Krankheiten stellen (angelehnt an das Propädeutikum und andere Module) mögliche Themen der OSCE-Station Diagnosemitteilung dar. Neben Kurzanamnese, Untersuchungsergebnissen und Diagnose enthalten sie Therapieoptionen, so dass die Studierenden PEF [15] erproben können. Mittels eines vorab ausgefüllten Profilbogens und im Briefing vor den jeweiligen Praktikumsterminen haben die SP selbstverständlich Mitspracherecht zu den Krankheitsbildern, die sie bereit sind, zu spielen. Die letzte Einheit bietet unter Einbindung von SP Raum zur Wiederholung und zum Prüfungstraining. Je nach Bedarf sind die SP auf Anamneseerhebung und Diagnosemitteilung vorbereitet.

Das Praktikum wird 14-tägig von einer Vorlesung begleitet (acht Termine zu je einer Stunde), die den theoretischen Hintergrund vertieft (neben den bereits genannten Referenzen auch unter Bezug auf [16]) sowie klinisches Wissen u. a. im Rahmen von Patientenvorstellungen vermittelt. Videogestütztes Lernen ist Bestandteil des Praktikumskonzeptes, sowohl durch den Einsatz von Lehrvideos zur Analyse und Diskussion als auch im Einsatz zur Aufzeichnung und Auswertung von praktischen Übungen der Studierenden. Schriftliche Unterlagen werden den Studierenden in der Regel über die eLearning-Plattform ILIAS der MHH zur Verfügung gestellt.

Der vorliegende Artikel berichtet im Folgenden über die Ergebnisse der internen Evaluation des Praktikums im 
Tabelle 1: Überblick zu Inhalten und Methoden des Praktikums (Studienjahr 2009/10)

\begin{tabular}{|c|c|c|}
\hline & Inhalte & Methoden* \\
\hline 1. Termin & $\begin{array}{l}\text { Grundlagen ärztlicher } \\
\text { Gesprächsführung } 1 \\
\text { Struktur und Funktion des ärztlichen } \\
\text { Gespräches: Erstgespräch Anamnese- } \\
\text { erhebung und Diagnosemitteilung }\end{array}$ & $\begin{array}{l}\text { Impulsvortrag zur Einführung (Dozent) } \\
\text { Praktische Übungen in Kleingruppen } \\
\text { (videounterstützt) }\end{array}$ \\
\hline 2. Termin & $\begin{array}{l}\text { Grundlagen ärtlicher } \\
\text { Gesprächsführung } 2 \\
\text { Kommunikationsaxiome und -modelle } \\
\text { Verbale und nonverbale Kommunikation } \\
\text { Basisfertigkeiten: Aktives Zuhören und } \\
\text { vertrauensvolle Arzt-Patienten- } \\
\text { Beziehung einleiten und aufbauen } \\
\text { Mehrdimensionalität von Äußerungen }\end{array}$ & $\begin{array}{l}\text { Impulskurzvorträge zur Theorie } \\
\text { (Dozent) } \\
\text { Praktische Übungen in Kleingruppen } \\
\text { (videounterstützt) } \\
\text { Rollenspiele unter den Studierenden }\end{array}$ \\
\hline 3. Termin & $\begin{array}{l}\text { Anamneseerhebung } 1 \\
\text { Biopsychosoziale Anamnese nach } \\
\text { Calgary-Cambridge Guides [4], [11], } \\
\text { [12] und FüeßI \& Middecke [13] }\end{array}$ & $\begin{array}{l}\text { Auswertung des Selbststudiumsanteils } \\
\text { zur "Anamnese einer älteren Person" } \\
\text { Impulskurzvorträge Theorie (Dozent) } \\
\text { Praktische Übungen (Rollenspiele) in } \\
\text { Kleingruppen }\end{array}$ \\
\hline 4. Termin & $\begin{array}{l}\text { Anamneseerhebung } 2 \\
\text { Biopsychosoziale Anamnese nach } \\
\text { Calgary-Cambridge Guides [4], [11], } \\
\text { [12] und FüeßI \& Middecke [13] }\end{array}$ & $\begin{array}{l}\text { Rollenspiele mit Simulationspatienten } \\
\text { (Simulation von Erstkontakten) }\end{array}$ \\
\hline 5. Termin & $\begin{array}{l}\text { Diagnosemitteilung } 1 \\
\text { Mitteilung einer schwerwiegenden } \\
\text { Diagnose nach Calgary-Cambridge } \\
\text { Guides [4], [11], [12] und SPIKES [14] }\end{array}$ & $\begin{array}{l}\text { Impulskurzvorträge zur Theorie } \\
\text { (Dozent) } \\
\text { Praktische Übungen (Rollenspiele) in } \\
\text { Kleingruppen }\end{array}$ \\
\hline 6. Termin & $\begin{array}{l}\text { Diagnosemitteilung } 2 \\
\text { Mitteilung einer schwerwiegenden } \\
\text { Diagnose nach Calgary- Cambridge } \\
\text { Guides [4], [11], [12] und SPIKES [14] }\end{array}$ & $\begin{array}{l}\text { Rollenspiele mit Simulationspatienten } \\
\text { (Simulation von Erstkontakten) }\end{array}$ \\
\hline 7. Termin & $\begin{array}{l}\text { Wiederholung („Prüfungstraining“) } \\
\text { Anamneseerhebung und } \\
\text { Diagnosemitteilung }\end{array}$ & $\begin{array}{l}\text { Rollenspiele mit Simulationspatienten } \\
\text { (Simulation von Erstkontakten) }\end{array}$ \\
\hline
\end{tabular}

Studienjahr 2009/10. Dabei stehen im Hinblick auf dessen zentrale Lernziele der Lernbedarf der Studierenden und ihre Kompetenzen zu Beginn des Praktikums (TO) sowie der Lernfortschritt zum Ende des Praktikums (T1) im Mittelpunkt. Die zentrale Fragestellung lautet, ob die Studierenden ihre Kompetenzen zu T1 höher einschätzen als zu TO. Darüber hinaus werden zentrale Methoden wie der Einsatz der SP evaluiert. Alle Daten wurden über Selbstangaben erfasst.

\section{Methode}

\section{Fragebogen}

Die eigens für die Lernziele entwickelten Fragebogen orientierten sich an den Kompetenzen, die an den OSCEStationen Anamneseerhebung und Diagnosemitteilung geprüft werden. Der TO-Fragebogen beinhaltet sieben Items zur Selbsteinschätzung, und zwar in Anlehnung an [17] Items zum eigenen Wissen (,to know how“) und Zutrauen in die eigene Handlungsfähigkeit („to show how“) bezüglich Anamneseerhebung und Diagnosemitteilung, jeweils ein „to show how“-Item zur Selbstsicherheit im Patientenkontakt und zur Abstimmung von Therapieoptionen mit Patienten (PEF) sowie ein Item zum Bewusst- sein über die eigenen Stärken und Schwächen in der Gesprächsführung (siehe Tabelle 2). Parallel enthält der Bogen eine Spalte zum jeweiligen Lernbedarf. AbschlieBend bietet er ein Freitextfeld, in dem nach bereits vorhandenen Erfahrungen mit Patienten im medizinischen Bereich (außer Pflegepraktikum) gefragt wird. Die Angaben zu Alter und Geschlecht komplettieren den Bogen. Der Abschlussfragebogen (T1) greift die sieben kompetenzbezogenen Fragen für den Prä-Post-Vergleich wieder auf und enthält darüber hinaus Aussagen zur Bewertung des Einsatzes der SP, der Videounterstützung und dem Ausmaß der Theorie- und Praxisphasen. Für alle Items stand eine Likertskala von 1 (stimmt gar nicht) bis 5 (stimmt voll und ganz) zur Verfügung. Zusätzlich erhielten die Studierenden im T1-Fragebogen in Freitextfeldern die Möglichkeit, aus ihrer Sicht besonders Positives zu benennen und Optimierungsvorschläge zu formulieren.

\section{Stichprobe und Vorgehen}

Insgesamt haben im Studienjahr 2009/10 285 Studierende am Praktikum teilgenommen. Der T0-Fragebogen wurde in der ersten Praktikumseinheit ausgegeben und von 267 Studierenden ausgefüllt (Teilnahmerate: 93,7\%). Von diesen füllten 180 Studierende den T1-Fragebogen am Ende des Praktikums aus (dies entspricht 63,2\% der 
Ende des Sommertrimesters einige der Studierenden den letzten Termin als „ihren“ Fehltermin genommen haben (zur Frage der dadurch möglicherweise induzierten Selektivität der T1-Teilnahme vgl. die durchgeführte DropoutAnalyse, s. u.).

\section{Auswertungsmethoden}

Nach einer Beschreibung der Stichprobe nach Geschlecht, Alter und Erfahrung mit Patienten sowie einer Analyse des Teilnahmeausfalls von T0 zu T1 (Dropoutanalyse) wurden mögliche Unterschiede im Lernbedarf zu TO, in den Kompetenzeinschätzungen zu TO sowie den Bewertungen der Methoden zu T1 jeweils mittels Varianzanalyse mit Messwiederholung getestet. Die sieben bzw. vier Items wurden dabei jeweils als Messwiederholungsfaktor definiert. Bei allen drei Varianzanalysen wurde die Sphärizitäts-Annahme mit dem Mauchly's Sphericity Test überprüft. Dieser Test war jeweils signifikant ( $p<.0001)$, und die Annahme damit verletzt (es zeigten sich also jeweils ungleich große Varianzen der Differenzen aller Paare von Messwerten). Da Epsilon in allen Analysen > .75 war (Lernbedarf: .87; Kompetenzeinschätzungen: .91; Bewertungen der Methoden: .89), wurde die HuynhFeldt-Korrektur angewendet und die entsprechenden Freiheitsgrade und $\mathrm{p}$-Werte berichtet. Allerdings ergaben sich dadurch weder im Vergleich zum Modell unter Annahme der Sphärizität noch zum multivariaten Ansatz divergierende Schlussfolgerungen Der Lernfortschritt bezüglich der einzelnen Kompetenzen (Längsschnittanalysen T1T0) wurde jeweils mittels T-Test für verbundene Stichproben getestet (alle Analysen: SPSS/PASW Statistics Version 18).

\section{Ergebnisse}

\section{Beschreibung der Stichprobe und Dropoutanalyse}

Tabelle 3 beschreibt die Stichprobe zu TO nach Geschlecht und Alter bzw. der Erfahrung mit Patienten. Mehr als zwei Drittel der Studierenden sind Frauen (68,8\%). Auffällig ist, dass sie eine höhere Altersvariabilität als die männlichen Studenten aufweisen (Standardabweichung=3,6, vs. 2,4), während sie sich im Mittel nicht signifikant unterscheiden. Eigene Erfahrung mit Patienten außerhalb des Pflegepraktikums haben insgesamt knapp die Hälfte der Studierenden (49,8\%, wobei dieser Anteil bei den Männern höher als bei den Frauen - 56,4\% vs. $45,7 \%$-, allerdings nicht signifikant).

Die im Hinblick auf die Längsschnittanalysen durchgeführte Dropoutanalyse ergab folgende Ergebnisse. Von den Studierenden, für die zu beiden Messzeitpunkten Daten vorlagen, waren 67\% Frauen, während dieser Anteil in der Gruppe ohne Daten zu T1 mit 72,7\% höher lag, allerdings statistisch nicht signifikant $\left(\mathrm{Chi}^{2}=0.81, \mathrm{p}=.368\right)$. Ebenfalls nicht signifikant waren der Altersunterschied zwischen beiden Gruppen ( $M=22,8$ vs. $22,9, F_{(1,257)}=0.02$, $\mathrm{p}=.899)$ und die Häufigkeit von Erfahrung mit Patienten (46,9\% vs. 55.8\%, Chi²=1.85, p=.174). Beim Lernbedarf ergab sich ein signifikanter Unterschied in Gestalt eines höheren Wertes in der Gruppe mit Daten zu TO und T1 bezüglich der Einschätzung eigener Stärken und Schwächen in der ärztlichen Gesprächsführung ( $M=4,1$ vs. 3,8, $\left.F_{(1,260)}=3.99, p=.047\right)$. In den Selbsteinschätzungen zu den eigenen Kompetenzen zu T0 zeigten sich Unterschiede in dem Zutrauen, eine Anamnese erheben zu können $(\mathrm{M}=2,5$ in der Gruppe mit Daten zu T0 und T1 vs. 2,2, $\left.F_{(1,264)}=6.19, p=.013\right)$, dem Wissen, worauf bei einer verständlichen Diagnosemitteilung zu achten ist (3,1 vs. 2,7, $\left.F_{(1,266)}=11.43, p=.001\right)$, und dem Zutrauen, eine schwerwiegende Diagnose mitteilen zu können (2,7 vs. 2,4, $\left.F_{(1,266)}=4.63, p=.032\right)$.

\section{Subjektiver Lernbedarf zu T0}

Wie Abbildung 1 zeigt, sehen die Studierenden für sich im Mittel einen erkennbaren Lernbedarf hinsichtlich der Lernziele des Praktikums. Zugleich gibt es signifikante Unterschiede $\left(F_{(5,247)}=23,4, p<.0001\right)$. Der im Mittel höchste Bedarf zeigt sich bei den „to show how“-Items bezüglich Anamneseerhebung und Diagnosemitteilung (jeweils $M=4,4$ [Median=5, hier nicht gezeigt]), gefolgt von dem Item zur PEF (Median=5) und den beiden entsprechenden Wissensitems (Median=4). Die Werte für das sichere Zugehen auf neue Patienten und die Einschätzung eigener Stärken und Schwächen bezüglich ärztlicher Gesprächsführung fallen - auf ähnlichem Niveau - etwas geringer aus ( $M=4,0$ bzw. 3,9 und Median=4).

\section{Selbsteinschätzungen der Kompetenzen zu TO und T1 und Lernfortschritt (Vergleich T1-T0)}

Wie Abbildung 2 zunächst für T0 zeigt, gab es die im Mittel niedrigsten Selbsteinschätzungen der eigenen Kompetenzen bei den „to show how“-Items zur Anamneseerhebung und Diagnosemitteilung ( $M=2,2$ bzw. 2,4 [Median=2, hier nicht gezeigt]), gefolgt von den entsprechenden Wissensitems (Median=2 bzw. 3) sowie der Frage zur PEF (Median=3). Die Werte für sicheres Zugehen auf neue Patienten und die eigenen Stärken und Schwächen fallen etwas höher aus ( $M=3,4$ bzw. 3,1, Median=3). Damit verhalten sich die Selbsteinschätzungen der Kompetenzen invers zum jeweiligen Lernbedarf (s. o.). Die Unterschiede sind statistisch signifikant $\left(F_{(5,429)}=48,6, p<.0001\right)$.

Die „T1-T0“-Vergleiche zeigen, dass sich bei allen sieben Lernzielen signifikante Fortschritte ergaben. Die größten Unterschiede gab es bei den anamnesespezifischen Items („to know how“: Mittelwertsdifferenz = 1,7, „to show how“: $1,8, p<.0001$ bei sämtlichen Tests) und beim „to know how“-Item zur Diagnosemitteilung $(1,6)$. Es folgen die Selbsteinschätzungen zur Mitteilung einer Diagnose $(1,4)$, PEF (1,2), Einschätzung der eigenen Stärken und Schwächen $(1,0)$ sowie zum sicheren Zugehen auf neue 
Tabelle 3: Beschreibung der Stichprobe ( $\mathrm{N}=267)$ nach Geschlecht, Alter und Erfahrung mit Patienten*

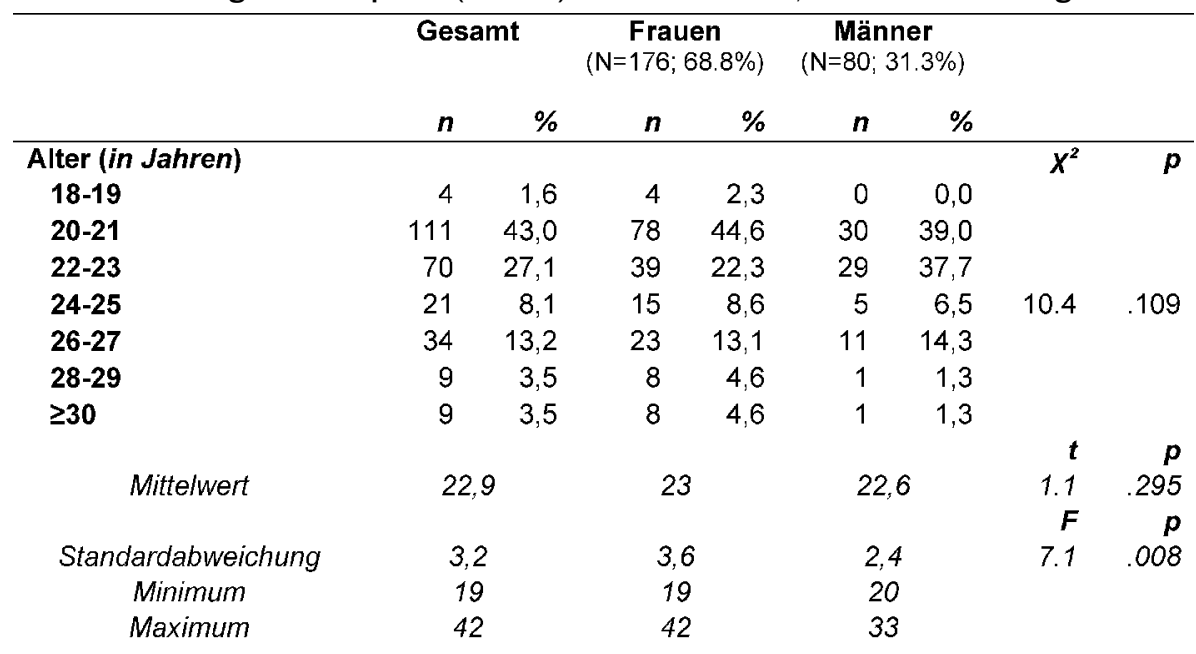

Erfahrung mit Patienten

\begin{tabular}{lrrrrrrrr}
$\begin{array}{l}\text { (außer Pflegepraktikum) } \\
\text { ja }\end{array}$ & 130 & 49,8 & 79 & 45,7 & 44 & 56,4 & $\boldsymbol{X}^{2}$ & $p$ \\
nein & 131 & 50,2 & 94 & 54,3 & 34 & 43,6 & 2.5 & .115 \\
\hline
\end{tabular}

* Abweichungen von $\mathrm{N}=267$ aufgrund fehlender Werte

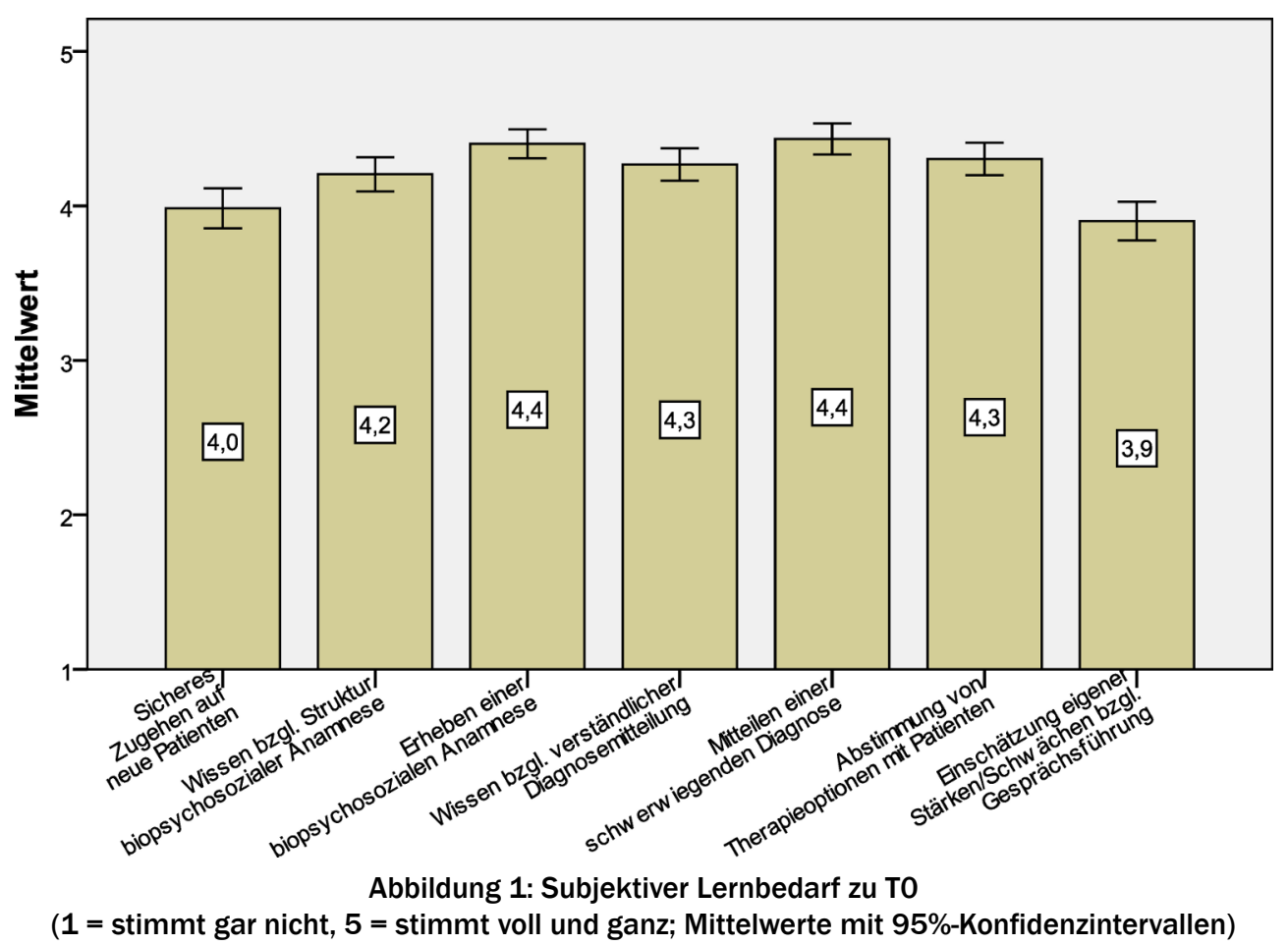

Patienten $(0,7)$. Zugleich ergab eine nach Ausgangsniveau stratifizierte Auswertung, dass Studierende, die sich zu T0 auf der jeweiligen Skala durch den Wert 1 oder 2 beschrieben hatten, sich über alle Items im Mittel um 2,2 Punkte verbessert hatten, solche mit einer 3 um 1,1 Punkte, und solche mit 4 oder 5 um 0,1 (hier nicht gezeigt).

Wie Abbildung 3 zeigt, haben die Studierenden den Einsatz der SP im Mittel am besten bewertet $(M=4,8)$, wobei $87 \%$ „stimmt voll und ganz“ ausgewählt hatten. Die Theorie- und Praxiseinheiten wurden auf einem Niveau und etwas weniger positiv als die SP bewertet ( $M=4,2$ und 4,0). Der Einsatz von Videotechnik zur Aufnahme von
Rollenspielen wurde im Vergleich dazu von mehr Studierenden als wenig positiv bewertet $(M=3,5)$. Die Unterschiede waren statistisch signifikant $\left(F_{(2,673)}=64.9, p<.0001\right)$. Der Median der Items (außer dem bezüglich der SP) lag jeweils bei 4 . 


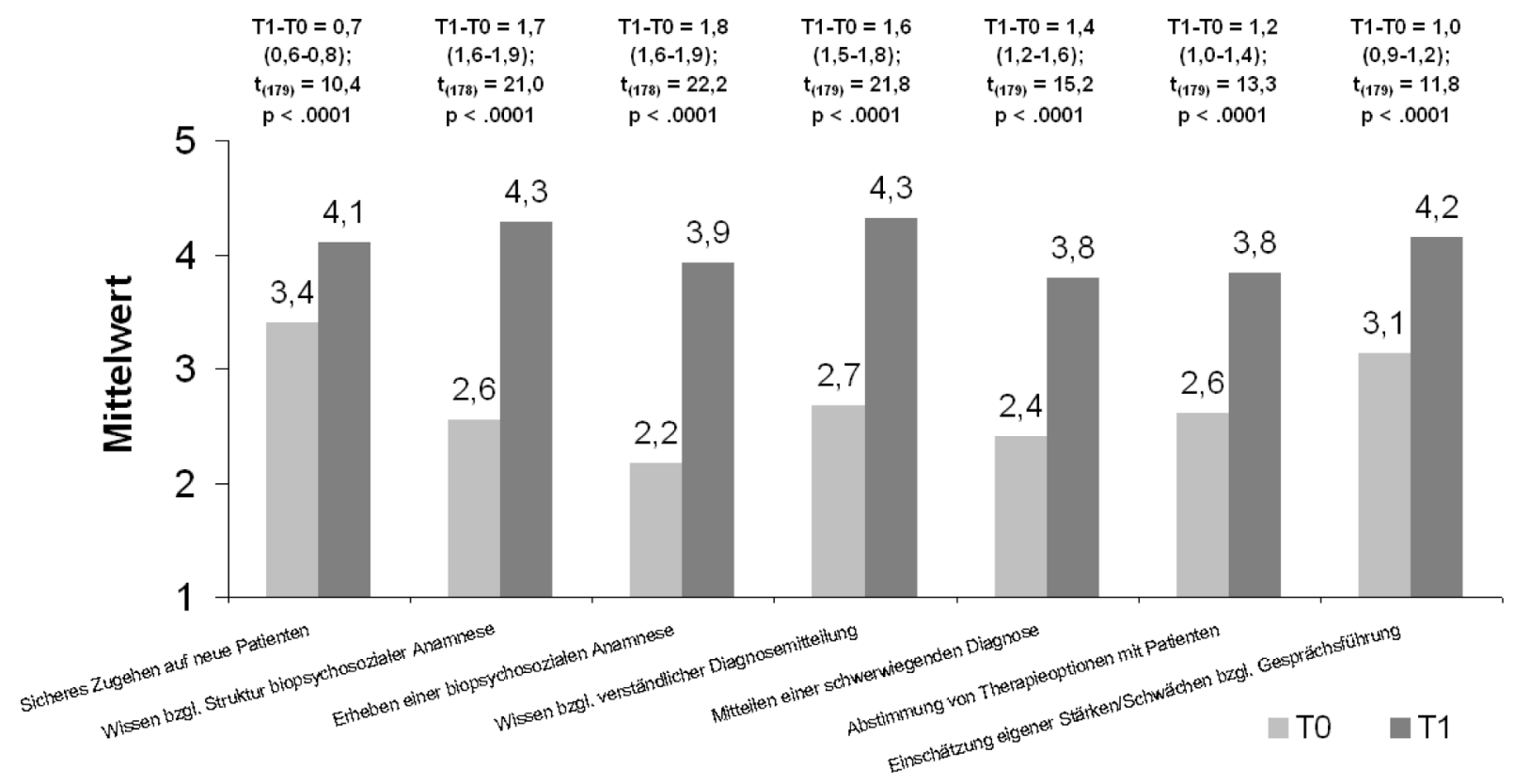

Abbildung 2: Selbsteinschätzungen der Kompetenzen zu T0 und T1 und Lernfortschritt (Vergleich T1-TO) ( 1 = stimmt gar nicht, 5 = stimmt voll und ganz; Mittelwertsdifferenzen mit 95\%-Konfidenzintervallen; t-Tests für abhängige Stichproben; $\mathrm{N}=180$, davon abweichende Freiheitsgrade aufgrund fehlender Werte)

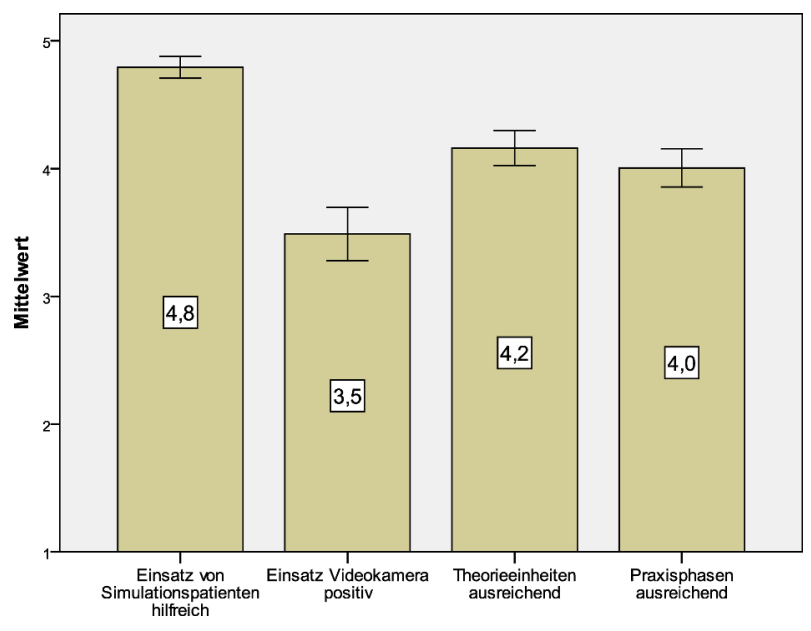

Abbildung 3: Bewertung verschiedener Lehrmethoden (zu T1) (1 = stimmt gar nicht, 5 = stimmt voll und ganz; Mittelwerte mit 95\%-Konfidenzintervallen)

\section{Diskussion}

Zusammenfassend ist das Gesprächsführungspraktikum im 2. HannibaL-Studienjahr aus Sicht der Studierenden bzgl. aller zentralen Lernziele mit bedeutsamen Lernfortschritten assoziiert. Dies gilt vor allem für Anamneseerhebung und Diagnosemitteilung als den zentralen Themen, und zwar sowohl im Sinne des „to know how“ als auch des „to show how“. Dabei zeigte die Erhebung einer biopsychosozialen Anamnese den stärksten Lernfortschritt, gefolgt von der patientenorientierten Mitteilung einer Diagnose, PEF, der Einschätzung der eigenen Stärken und Schwächen und dem Zutrauen, sicher auf neue Patienten zugehen zu können. Dabei hatten die zwei letzteren zu Beginn den geringsten Lernbedarf und die besten Selbsteinschätzungen gezeigt. Weiterhin hatten sich Studierende mit anfangs eher schlechten Selbsteinschätzungen („1“ oder „2“ auf 5-Punkt-Skalen) über alle Lernziele im Mittel um 2,2 Punkte verbessert, solche auf mittlerem Niveau um 1,1 Punkte, und solche mit guten oder optimalen T0-Werten um 0,1 Punkte. Bei der Methodenbewertung schnitt der SP-Einsatz am positivsten ab, gefolgt von der Menge an Theorie- und Praxisphasen sowie dem Einsatz von Videofeedback.

Bevor diese Ergebnisse hinsichtlich ihrer möglichen Implikationen für die Vermittlung sozial-kommunikativer Kompetenzen im Medizinstudium diskutiert werden, sind einige, vor allem methodische Einschränkungen der Studie zu nennen. Zunächst handelt es sich um einen Prä-Post-Vergleich und damit lediglich um ein quasi-experimentelles Design [18]. Es war also im Hinblick auf das Praktikum als Intervention kein (randomisierter) Vergleich mit einer Kontrollgruppe (z. B. mit „traditioneller“ Ausbildung in der Gesprächsführung) möglich, der sicherlich ein Gewinn für die Einordnung der Ergebnisse wäre. Dies ist allerdings im Rahmen der vorliegenden Thematik nicht ungewöhnlich [19], [20], [21], [22], [23], [24], [25], [26], [27], [28], [29], und gleichwohl erlaubt der Vorher-Nachher-Vergleich die Darstellung eines Kompetenzzuwachses. Zugleich ist zu fragen, welche externen Einflussfaktoren aus dem Umfeld der Studierenden diese spezifischen Gesprächsführungskompetenzen verbessert haben sollten. Dennoch zeigt eine frühere Studie mit Kontrollgruppe, in der in einer Experimentalgruppe mit OSCE-Training auf einigen Parametern schlechtere Ergebnisse als in einer Kontrollgruppe aus einem früheren Semester gefunden worden waren [30], dass kausalen Interpretationen der Praktikumseffekte in der vorliegenden Studie mit Bedacht begegnet werden sollten.

Zweitens basiert die Abbildung der Lernfortschritte ausschließlich auf Selbsteinschätzungen der Studierenden. Eine objektivierende Erfassung durch Performanzbewer- 
tungen im Praktikum war didaktisch nicht erwünscht, und eine Verknüpfung mit den OSCE-Noten aus organisatorischen Gründen nicht ohne Weiteres möglich (Letzteres ist seitens der Autoren für zukünftige Studienjahre beabsichtigt). Streng genommen wurden durch die lernzielbezogenen Selbsteinschätzungen also keine Kompetenzen erfasst (wozu Beobachtungsbogen, SP-Befragungen oder theoretische Prüfungen notwendig wären [31]), sondern entsprechende Selbstwirksamkeitserwartungen (SWE). Generell sind SWE als Überzeugungen definiert, fähig zu sein, zielführendes Verhalten selbst ausführen zu können [32]. Je selbstwirksamer sich eine Person erlebt, desto wahrscheinlicher wird sie ein Verhalten zukünftig auch ausführen (hier also Fähigkeiten zeigen) können. Daher wird im vorliegenden Fall die genannte Einschränkung zumindest z. T. dadurch kompensiert, dass positive SWE auch bei Medizinstudierenden eine wichtige vermittelnde Größe zwischen übungsinduzierten Fähigkeiten und der Performanz z. B. in einer OSCE darstellen [33]. Somit kann davon ausgegangen werden, dass die gestiegenen SWE zu einer verbesserten Performanz beitragen haben - die auch hätte dargestellt werden können, wenn entsprechende Messungen verfügbar gewesen wären (vgl. auch [34]).

Drittens wurde ein selbstentwickelter Fragebogen eingesetzt, um die konkreten Lernziele des Praktikums möglichst genau abzubilden. Somit ist zum Einen die Vergleichbarkeit zu anderen Studien eingeschränkt, und alternativ wäre z. B. eine Adaptation der Skalen von Parle et al. [35] ähnlich wie durch Ammentorp et al. [36] möglich gewesen (wobei diese allerdings den Transfer von der Fort- auf die Ausbildung hätte leisten müssen). Zum Anderen müssen weitere Analysen und Erfahrungen mit den Skalen zeigen, ob die numerisch eher geringen Lernfortschritte bei den Studierenden mit hohen Selbsteinschätzungen ihrer Kompetenz zu TO Deckeneffekte darstellen bzw. ob durch modifizierte Antwortskalen auch in dieser Subgruppe deutlichere Veränderungen darstellbar wären.

Schließlich lag von 32,6\% der T0-Teilnehmer kein T1Fragebogen vor. Dabei ist wie erwähnt zu berücksichtigen, dass ein Fehltermin formal zulässig war und aufgrund der Prüfungsdichte am Ende des Sommertrimesters einige Studierende den letzten Termin als Fehltermin gewählt haben. Hier könnte vermutet werden, dass die T1-Teilnahme insofern selektiv war, dass besonders Studierende mit positiven Selbsteinschätzungen zugunsten anderer Prüfungen auf die letzte Praktikumseinheit verzichtet haben. Die Dropoutanalyse deutet allerdings darauf hin, dass dieser Effekt eher schwach ausgefallen ist. Zwar zeigten alle signifikanten Unterschiede in den TO-Kompetenzeinschätzungen zwischen T1-Teilnehmern und -Nichtteilnehmern (drei von sieben Items) höhere Werte bei den T1-Teilnehmern, jedoch ist das Niveau dieser Unterschiede (0,3-0,4 Skalenpunkte) eher kein Indiz für ein ausgeprägtes „Expertentum“ der T1-Teilnehmer. Zugleich hatten unter den T1-Teilnehmern auch die mit relativ guten Selbsteinschätzungen einen Lernfortschritt gezeigt, so dass - hätten alle T0-Teilnehmer zu T1 teilgenommen - die Zuwächse insgesamt im Mittel zumindest nicht durch negative Veränderungen reduziert worden wären. Da zugleich Geschlecht, Alter und Erfahrung nicht signifikant mit dem Dropout assoziiert waren, scheint dieser die interne Validität nicht schwerwiegend beeinträchtigt zu haben. Nichtsdestotrotz sind hier zukünftig organisatorische Maßnahmen erstrebenswert, um die T1-Teilnahmeraten zu erhöhen, z. B. eine entsprechende zusätzliche Onlineversion des T1-Fragebogens.

Insgesamt deuten die Ergebnisse der Studie darauf hin, dass das Praktikum bedeutsam zur Vermittlung sozialkommunikativer Kompetenzen der $\mathrm{MHH}$-Studierenden beiträgt. Es setzt an Lernbedarfen an, die seitens der Studierenden formuliert werden. Die Lernfortschritte, die sich in Selbsteinschätzungen ergeben haben, weisen sowohl auf die Angemessenheit der Inhalte und Methoden des Praktikums für die Studierenden als auch ihre Offenheit für diese hin. Dies ist nicht zuletzt im Hinblick auf die Platzierung des Praktikums im 2. Studienjahr relevant, da einige der Studierenden in den Einstiegsplena der Praktika die Befürchtung äußerten, für sorgsame Anamneseerhebungen und - vor allem - fundierte Diagnosemitteilungen über kein ausreichendes medizinisches Wissen zu verfügen. Da diese Befürchtung auch in den Freitextfeldern des Abschlussbogens nicht genannt worden war, ist anzunehmen, dass der Gesprächsführungsfokus des Praktikums erfolgreich deutlich gemacht werden konnte. Wiewohl die OSCE als Teil der Modulabschlussprüfungen gewiss ebenfalls motivierend wirkt, konnte den Studierenden u. E. durch den methodisch-didaktischen Aufbaus des Praktikums die notwendige Orientierung zum Gesamtkonzept und dessen Zielsetzung vermittelt werden, die ein strukturiertes, aufeinander aufbauendes und standardisiertes Lehren und Lernen ermöglichte.

Die besonders positive Bewertung der SP als didaktischmethodisches „Mittel“ deckt sich mit den Ergebnissen zahlreicher früherer Studien (vgl. zusammenfassend [4], [6], [7]) und den Angaben in den T1-Freitextfeldern, die von 53\% der Studierenden genutzt worden waren und bei denen sich 44 der 139 positiven Nennungen auf die SP bezogen. Sie ermutigt, diese seit 2008 auch auf das OSCE-vorbereitende Praktikum ausgebaute „Infrastruktur“ zu verstetigen und weiter zu optimieren, $u$. a. durch weitere Schulung der Feedbackkompetenz der SP [37] und Weiterentwicklung von Rollenstandards sowie weiterer qualitätssichernder Maßnahmen [38]. Auch das Fehlen von negativer Kritik zur interaktiven Methode des Rollenspiels, die hohe Anteile an Selbsterfahrung und Positionierung vor Mitstudierenden beinhaltet [39], im entsprechenden Freitextfeld des T1-Fragebogens spricht für das Praktikumskonzept. Durch Aufteilen der Zehnergruppen in Kleingruppen auch bei den SP-Terminen und das explizite Trainieren konstruktiver Rückmeldungen wurden die Studierenden mit dem Agieren vor der Gruppe von Anfang an vertraut gemacht.

Die signifikant weniger positiven Bewertungen der Quantität von Theorie- und Praxiseinheiten werden ebenfalls in den Freitextfeldangaben gespiegelt, in denen von insgesamt 73 Optimierungsvorschlägen 43 das Bedürfnis nach mehr Praxis und weniger Theorie benannten. 
Möglicherweise ist diese Balance vor allem hinsichtlich der Lernziele zur Übermittlung auch schwerwiegender Diagnosen noch nicht optimal gelungen, die als Thema erst ab dem fünften Praktikumstermin speziell aufgegriffen wurde. Dies hat im Rahmen der Qualitätssicherung und kontinuierlichen Weiterentwicklung des Praktikumskonzeptes im Nachlauf zum Studienjahr 2009/10 bereits zu einer konzeptionellen Veränderung geführt, die auf die konsequente Vermittlung der Grundlagen ärztlicher Gesprächsführung an den Themen Anamneseerhebung (im 1. Praktikumstermin) und Diagnosemitteilung (2. Termin) i. S. einer Themenzentrierung „von Anfang an“ hinausläuft.

Schließlich spiegelt die eher moderat positive Bewertung des Einsatzes von Videofeedback einen Optimierungsbedarf im Rahmen des Praktikumskonzeptes wider. Denn unabhängig davon, dass sich diese Methode als wertvoll bei der Vermittlung von Kommunikationskompetenzen erwiesen hat [4], [24], bedingt sie durch die Vorführung und Besprechung der Aufnahmen einen deutlichen Mehraufwand an Zeit. In diesem Zusammenhang ist unsere Erfahrung, dass die Studierenden in der Tendenz eher die Präferenz äußern, zuungunsten von Videoanalysen die Anzahl der Rollenspiele zu erhöhen. Hier ist es eine Aufgabe für die Weiterentwicklung des Praktikumskonzeptes, die Integration dieser Methode konsequenter zu gestalten und eine bessere Balance zwischen vertiefter Analyse und der Anzahl von Simulationen herzustellen. Zudem wären Infrastrukturen wie Beobachtungsräume mit entsprechender Ausrüstung (und verspiegelten Glaswänden wie beispielsweise im Studienhospital Münster $\left.{ }^{\circledR}\right)$ förderlich.

Im Übrigen wies eine weitere Auswertung der Freitextfelder auf weitere, „atmosphärische“ Hintergründe für den Erfolg des Praktikums hin. Die fachliche, pädagogische und persönliche Kompetenz des/r jeweiligen Dozenten/in wurde in 51 Nennungen honoriert. 14 Nennungen beschrieben die Gruppengröße von 10 Studierenden als optimal. Zudem wurden die Gelegenheit, aktives Erfahrungslernen mit den anderen unter Wahrung der eigenen Individualität zu erleben, und die geringe Schwelle auch für eher introvertierte Studierende, sich zu engagieren, positiv wahrgenommen. Noch mehr Partnerinterviews und Kleingruppenarbeit innerhalb der Zehnergruppen wurden in neun Nennungen eingefordert.

Auf eine nach Geschlecht und Erfahrung stratifizierte Darstellung der Ergebnisse wurde verzichtet, da die entsprechenden Auswertungen lediglich marginale Unterschiede ergaben. So zeigte sich bei den Prä-Post-Vergleichen mit dem Faktor Geschlecht lediglich bezüglich des sicheren Zugehens auf neue Patienten ein Interaktionseffekt dergestalt, dass die Männer zu TO geringfügig bessere Selbsteinschätzungen hatten ( $M=3,8$ vs. 3,2) und die Frauen über einen etwas deutlicheren Lernfortschritt berichteten $(0,8$ vs. 0,$5 ; p=.032)$. Alle anderen Tests blieben ebenso wie die bei den Methodenbewertungen ohne signifikante Ergebnisse. Auch beim Lernbedarf fielen alle Mittelwertsdifferenzen mit maximal 0,4 Punkten geringer als beim Lernfortschritt aus. Die Unterschiede deuten damit zwar auf eine teilweise leicht positivere Selbstsicht der männlichen Studierenden hin und sind mit stärker ausgeprägten Arztidealen bei weiblichen Medizinstudierenden [40] konsistent. Dennoch scheinen genderspezifische Aspekte in der vorliegenden Studierendenkohorte hinsichtlich der Aneignung kommunikativer Kompetenzen untergeordnet.

Insgesamt stellt die positive Evaluation des Gesprächsführungspraktikums aus unserer Sicht ein weiteres, empirisches Argument für ein jahrgangsübergreifenden Curriculum zu sozial-kommunikativen Kompetenzen und Arzt-Patient-Gesprächsführung auch an der MHH dar, das bestehende Angebote longitudinal [41] in einer Lernspirale integriert und weiterentwickelt. Langfristig könnte dies auch Evaluationen beinhalten, die die Auswirkungen eines solchen Curriculums auf die spätere Performanz der Studierenden bzw. postgradual Ärzte im praktischen Jahr bzw. im Berufsalltag analysiert (zumal solche Effekte nicht voraussetzungslos sind [42]).

Darüber hinaus schließen wir uns der Empfehlung an, hochschul- bzw. fakultätsübergreifende Aktivitäten nicht zuletzt im Hinblick auf valide und reliable Prüfungsmethoden zu stärken [43]. Dafür sprechen auch dadurch zu erwartende Effizienzsteigerungen. Diese sind nicht zuletzt deshalb bedeutsam, weil die Umsetzung von Lehrkonzepten wie dem hier evaluierten (das praxisorientiertes Lehren und Lernen in kleinen Gruppen von Studierenden und mittels SP ermöglicht) beträchtlichen personellen, organisatorischen und finanziellen Aufwand erfordert. Es ist plausibel anzunehmen, dass dieser Aufwand besonders dann nachhaltig durchzusetzen ist, wenn jahrgangsübergreifende Curricula in diesem Kompetenzbereich zum Standard werden. Optimalerweise basieren solche, möglichst interdisziplinär konzipierten Aktivitäten auf einem konsentierten Rahmenmodell. Eine Blaupause für ein solches Modell könnte das britische Konsensuspapier [44] darstellen. Es definiert zunächst Respekt als Basis effektiver klinischer Kommunikation und benennt mit den Stichworten Theorie und Evidenz, Aufgaben klinischer Kommunikation, spezifischen Themen wie z. B. Verhaltensveränderung, Medien sowie Kommunikation über den Patienten hinaus praktisch alle relevanten Kernthemen. Wir hoffen, dass die hier vorgelegte Evaluation eines curricularen Bausteines an der $\mathrm{MHH}$ zu diesem Gesamtprozess beitragen wird.

\section{Anmerkungen}

*APG-Lehrteam MHH (Arzt-Patient-GesprächsführungsLehrteam MHH): Karin Lange (Federführung), Ulrich Brinkmeier, Gundula Ernst, Angelika Kursch, Thomas von Lengerke, Thomas Schneller (Forschungs- und Lehreinheit Medizinische Psychologie); Siegfried Geyer (Federführung), Anja Löbel, Friederike Otto, Stefanie Sperlich (Forschungs- und Lehreinheit Medizinische Soziologie); Eva Hummers-Pradier, Carsten Kruschinski, Heidrun Lingner, Christiane Müller, Erika Penner (Institut für Allgemeinmedizin); Uwe Hartmann, Susanne Philippsohn, Lotta Winter 
(Arbeitsbereich Klinische Psychologie der Klinik für Psychiatrie, Sozialpsychiatrie und Psychotherapie); Burkard Jäger, Maike Möllenkamp, Angela Trieschmann (Klinik für Psychosomatik und Psychotherapie)

**Lehrverantwortlicher des Jahrgangsübergreifenden Curriculums Innere Medizin: Prof. Dr. med. Hermann Haller (als Studiendekan für Medizin und Aufbau- und Ergänzungsstudiengänge an der $\mathrm{MHH}$ federführend bei der Entwicklung von HannibaL); Modulverantwortliche Diagnostische Methoden: Dr. med. Saskia Merkel.

\section{Danksagung}

Wir danken den Studierenden des Jahrgangs des Modellstudiengangs HannibaL mit Studienbeginn 2008 für ihre Beteiligung an der Studie und damit für ihre Bereitschaft, neben den Datenerhebungen des Evaluationsbüros des Studiendekanat Medizin der MHH einen weiteren Fragebogen auszufüllen.

\section{Interessenkonflikt}

Die Autoren erklären, dass sie keine Interessenskonflikte in Zusammenhang mit diesem Artikel haben.

\section{Literatur}

1. Bundesministerium der Justiz. Approbationsordnung für Ärzte vom 27. Juni 2002 (BGBI. I S. 2405), die zuletzt durch Artikel 10 des Gesetzes vom 24. Juli 2010 (BGBI. I S. 983) geändert worden ist. Berlin: Bundesministerium der Justiz; 2010. Zugänglich unter/available from: http://www.gesetze-iminternet.de/_appro_2002/BJNR240500002.html

2. Hahn EG, Fischer MR. Nationaler Kompetenzbasierter Lernzielkatalog Medizin (NKLM) für Deutschland: Zusammenarbeit der Gesellschaft für Medizinische Ausbildung (GMA) und des Medizinischen Fakultätentages (MFT). GMS Z Med Ausbild. 2009;26(3):Doc35. DOI: 10.3205/zma000627

3. Barrows HS. Simulated patients in medical training. Can Med Assoc J. 1968;98(14):674-676.

4. Kurtz S, Silverman J, Draper J. Teaching and learning communicating skills in medicine. 2nd edition. Oxford: Radcliffe; 2004.

5. Fröhmel A, Burger W, Ortwein $\mathrm{H}$. Einbindung von Simulationspatienten in das Studium der Humanmedizin in Deutschland. Dtsch Med Wochenschr. 2007;132(11):549-554. http://dx.doi.org/10.1055/s-2007-970375

6. Rockenbauch K, Fabry G, Petersen C, Daig I, Philipp S. Der Einsatz von Schauspielpatienten in der Medizinischen Psychologie - allgemeiner Überblick und konkrete Umsetzungsbeispiele. Z Med Psychol. 2008;17(4):185-192.

7. Simmenroth-Nayda A, Chenot JF, Fischer Th, Scherer M, Stanske $\mathrm{B}$, Kochen MM. Mit Laienschauspielern das ärztliche Gespräch trainieren. Dtsch Arztebl. 2007;104(13):A847-852.

8. Bokken L, Rethans J-J, Jöbsis Q, Duvivier R, Scherpbier A, van der Vleuten C. Instructiveness of real patients and simulated patients in undergraduate medical education: a randomized experiment. Acad Med. 2010;85(1):148-154. http://dx.doi.org/ 10.1097/ACM.0b013e3181c48130
9. Watzlawick P, Beavin JH, Jackson DD. Menschliche Kommunikation: Formen, Störungen, Paradoxien. Bern: Huber; 1969.

10. Schulz von Thun F. Miteinander reden. Reinbek: Rowohlt; 2008.

11. Kurtz S, Silverman J, Benson J, Draper J. Marrying content and process in clinical method teaching: enhancing the CalgaryCambridge Guides. Acad Med. 2003;78(8):802-809. http:// dx.doi.org/10.1097/00001888-200308000-00011

12. Silverman J, Kurtz S, Draper J. Skills for communicating with patients. 2nd edition. Oxford: Radcliffe; 2005.

13. FüeßI HS, Middeke M. Anamnese und klinische Untersuchung. 4. Auflage. Stuttgart: Thieme; 2010.

14. Baile W, Buckman R, Lenzi R, Glober G, Beale EA, Kudelka AP. SPIKES-A six-step protocol for delivering bad news: application to the patient with cancer. Oncologist. 2000;5(4):302-311. http://dx.doi.org/10.1634/theoncologist.5-4-302

15. Scheibler F, Janssen C, Pfaff H. Shared decision making: ein Überblicksartikel über die internationale Forschungsliteratur. Soz Praventivmed. 2003;48(1):11-23. http://dx.doi.org/ $10.1007 / \mathrm{s} 000380300002$

16. Buser K, Schneller T, Wildgrube K. KLB Medizinische Psychologie - Medizinische Soziologie. 6. Auflage. München: Elsevier/Urban \& Fischer; 2007.

17. Miller GE. The assessment of clinical skills/competence/performance. Acad Med. 1990;65(9 Suppl):S63-67. http://dx.doi.org/10.1097/00001888199009000-00045

18. Mohr LB. Impact analysis for program evaluation. Chicago, II: Dorsey Press; 1988.

19. Bahrs O. Kommunikationsförderung in der Vor-Klinik - Kursus der Medizinischen Psychologie und Medizinischen Soziologie. Z Med Psychol. 2010;19(2):97-101.

20. Brenk-Franz K, Kubieziel H, Schulz S, Gensichen J.

Simulationspatienten in der allgemeinmedizinischen Lehre: Das Jenaer Programm - eine Pilotstudie. Z Allg Med. 2010;86(4):152158.

21. Fischbeck S, Mauch M, Leschnik E, Laubach W. Entwicklung und Evaluation einer OSCE für die Überprüfung kommunikativer ärztlicher Kompetenz im Kursus der Medizinischen Psychologie und Medizinischen Soziologie. Z Med Psychol. 2010;19(2):9496.

22. Fischer T, Chenot J-F, Kleiber C, Kochen MM, Simmenroth-Nayda A, Staats H, Hermann-Lingen C. Kurs „ärztliche Basisfähigkeiten“ - Evaluation eines primärärztlichen orientierten Unterrichtskonzeptes im Rahmen der neuen Approbationsordnung. GMS Z Med Ausbild. 2005;22(3):Doc59. Zugänglich unter/available from: http://www.egms.de/de/ journals/zma/2005-22/zma000059.shtml

23. Koerfer A, Köhle K, Obliers R, Sonntag B, Thomas W, Albus C. Training und Prüfung kommunikativer Kompetenz. Aus- und Fortbildungskonzepte zur ärztlichen Gesprächsführung. Gesprächsforschung. 2008;9:34-78. Zugänglich unter/available from: http://www.gespraechsforschung-ozs.de/heft2008/agkoerfer.pdf

24. Kopecky-Wenzel M, Maier EM, Muntau AC, Reinhardt D, Frank R. Überbringen schlechter Nachrichten - videogestützte Trainingseinheit für Medizinstudenten. Z Kinder Jugendpsychiatr Psychother. 2009;37(2):139-144. http://dx.doi.org/10.1024/ 1422-4917.37.2.139 
25. Mitzkat A, Schulz C, Kasenda B, Langer T, Schnell MW. „ARZT IM GANZEN SPREKTRUM“: Die INTEGRIERTEN CURRICULA der Medizinerausbildung an der Universität Witten/Herdecke Rückblick auf sechs Jahre Lehre im Hinblick auf Praxisorientiertung und theoretische Vorgaben. GMS Z Med Ausbild. 2006;23(4):Doc66. Zugänglich unter/available from: http://www.egms.de/static/de/journals/zma/2006-23/ zma000285.shtml

26. Petersen C, Busche W, Bergelt C, Huse-Kleinstoll G. Kommunikationstraining als Teil des Medizinstudiums: Ein Modellversuch. GMS Z Med Ausbild. 2005;22(1):Doc08. Zugänglich unter/available from: http://www.egms.de/static/ de/journals/zma/2005-22/zma000008.shtml

27. Philipp S, Merten K. Erfahrungen mit Standardisierten Patienten im neuen Lehrkonzept zur Vermittlung kommunikativer Kompetenzen im Fach Medizinische Psychologie - das Jenaer Modell. Z Med Psychol. 2006;15(1):11-19.

28. Pucher-Matzner I, Gleiss A, Schmidts M, Frischenschlager O. Die Checkliste PD „Professionelles ärztliches Kommunikationsverhalten " in Unterricht und Evaluation kommunikativer Fertigkeiten im Medizinstudium. GMS Z Med Ausbild. 2006;23(4):Doc68. Zugänglich unter/available from: http://www.egms.de/static/de/journals/zma/2006-23/ zma000287.shtml

29. Wiesmann U, Niehörster G, Hannich H-J. Medizinpsychologische Lehre innovativ gestalten: Förderung von Kommunikations- und Forschungskompetenzen in der vorklinischen Ausbildung. Z Med Psychol. 2009;18(2):88-96.

30. Kopp V, Schewe S. Kann durch Training Anamnese und Klinische Untersuchung vermittelt werden? GMS Z Med Ausbild. 2005;22(1):Doc15. Zugänglich unter/available from: http:// www.egms.de/static/de/journals/zma/2005-22/ zma000015.shtml

31. Duffy FD, Gordon GH, Whelan G, Cole-Kelly K, Frankel R; All Participants in the American Academy on Physician and Patient's Conference on Education and Evaluation of Competence in Communication and Interpersonal Skills. Assessing competence in communication and interpersonal skills: the Kalamazoo II Report. Acad Med. 2004;79(6):495-507. http://dx.doi.org/ 10.1097/00001888-200406000-00002

32. Bandura A. Self-efficacy: the exercise of control. New York: Freeman; 1997.

33. Mavis B. Self-efficacy and OSCE performance among second year medical students. Adv Health Sci Educ. 2001;6(2):93-102. http://dx.doi.org/10.1023/A:1011404132508

34. Schultz J-H, Schönemann J, Lauber H, Nikendei C, Herzog W, Jünger J. Einsatz von Simulationspatienten im Kommunikationsund Interaktionstraining für Medizinerinnen und Mediziner (MediKIT): Bedarfsanalyse - Training - Perspektiven. Gruppendynamik Organisationsberatung. 2007;38(1):7-23. http://dx.doi.org/ $10.1007 / \mathrm{s} 11612-007-0002-\mathrm{y}$

35. Parle M, Maguire P, Heaven C. The development of a training model to improve health professionals' skills, self-efficacy and outcome expectancies when communicating with cancer patients. Soc Sci Med. 1997;44(2):231-240. http://dx.doi.org/10.1016/ S0277-9536(96)00148-7

36. Ammentorp J, Sabroe S, Kofoed P-E, Mainz J. The effect of training in communication skills on medical doctors' and nurses' self-efficacy: a randomized controlled trial. Patient Educ Couns. 2007;66(3):270-277. http://dx.doi.org/10.1016/ j.pec.2006.12.012

37. Bokken L, Linssen T, Scherpbier A, van der Vleuten C, Rethans $\mathrm{JJ}$. Feedback by simulated patients in undergraduate medical education: a systematic review of the literature. Med Educ. 2009;43(3):202-210. http://dx.doi.org/10.1111/j.13652923.2008.03268.x
38. Cleland JA, Abe K, Rethans J-J. The use of simulated patients in medical education: AMEE Guide No 42. Med Teach. 2009;31(6):477-486. http://dx.doi.org/10.1080/ 01421590903002821

39. Dieckmann P, Rall M, Eich C, Schnabel K, Jünger J, Nikendei C. Rollenspiel als wesentliches Element bei Simulationsverfahren in der Medizin. Z Evid Fortbild Qual Gesundhwes. 2008;102(10):642-647. http://dx.doi.org/10.1016/ j.zefq.2008.11.020

40. Schrauth M, Kowalski A, Weyrich P, Begenau J, Werner A, Zipfel S, Nikendel C. Selbstbild, Arztbild und Arztideal: Ein Vergleich Medizinstudierender 1981 und 2006. Psychother Psych Med. 2009;59(12):446-453. http://dx.doi.org/10.1055/s-00291202343

41. Bachmann C, Hölzer H, Dieterich A, Fabry G, Langewitz W, Lauber $\mathrm{H}$, Ortwein H, Pruskil S, Schubert S, Sennekamp M, SimmenrothNayda A, Silbernagel W, Scheffer S, Kiessling C. Longitudinales, bologna-kompatibles Modell-Curriculum „Kommunikative und soziale Kompetenzen“: Ergebnisse eines interdisziplinären Workshops deutschsprachiger medizinischer Fakultäten. GMS Z Med Ausbild. 2009;26(4):Doc38. DOI: 10.3205/zma000631

42. Stimmel B, Cohen D, Fallar R, Smith L. The use of standardised patients to assess clinical competence: does practice make perfect? Med Educ. 2006;40(5):444-449 http://dx.doi.org/ 10.1111/j.1365-2929.2006.02446.x

43. Kiessling C, Dieterich A, Fabry G, Hölzer H, Langewitz W, Mühlinghaus I, Pruskil S, Scheffer S, Schubert S. Basler Consensus Statement „Kommunikative und soziale Kompetenzen im Medizinstudium “: Ein Positionspapier des GMA-Ausschusses Kommunikative und soziale Kompetenzen. GMS Z Med Ausbild. 2008;25(2):Doc83. Zugänglich unter/available from: http:// www.egms.de/static/de/journals/zma/2008-25/ zma000567.shtml

44. von Fragstein M, Silverman J, Cushing A, Quilligan S, Salisbury $\mathrm{H}$, Wiskin C; UK Council for Clinical Communication Skills Teeaching in Undergraduate Medical Education. UK consensus statement on the content of communication curricula in undergraduate medical education. Med Educ. 2008;42(11):1100-1107. http://dx.doi.org/10.1111/j.13652923.2008.03137.x

\section{Korrespondenzadresse:}

PD Dr. phil. Thomas von Lengerke

Medizinische Hochschule Hannover, Forschungs- und Lehreinheit Medizinische Psychologie (OE 5430), Carl-Neuberg-Straße 1, 30625 Hannover, Deutschland, Tel.: +49 (0)511/532-4445, Fax: +49 (0)511/532-4214 lengerke.thomas@mh-hannover.de

\section{Bitte zitieren als}

von Lengerke T, Kursch A, Lange K, APG-Lehrteam MHH*. Das Gesprächsführungspraktikum im 2. Studienjahr des Modellstudiengangs HannibaL: Eine Evaluation mittels Selbsteinschätzungen der Studierenden. GMS Z Med Ausbild. 2011;28(4):Doc54.

DOI: 10.3205/zma000766, URN: urn:nbn:de:0183-zma0007668

Artikel online frei zugänglich unter

http://www.egms.de/en/journals/zma/2011-28/zma000766.shtml

Eingereicht: 27.01.2011

Überarbeitet: 10.08.2011

Angenommen: 08.09.2011

Veröffentlicht: 15.11.2011 
Copyright

(C2011 von Lengerke et al. Dieser Artikel ist ein Open Access-Artikel und steht unter den Creative Commons Lizenzbedingungen

(http://creativecommons.org/licenses/by-nc-nd/3.0/deed.de). Er darf vervielfältigt, verbreitet und öffentlich zugänglich gemacht werden,

vorausgesetzt dass Autor und Quelle genannt werden. 


\title{
The communication skills course for second year medical students at Hannover Medical School: An evaluation study based on students' self-assessments
}

\begin{abstract}
In the model medical curriculum HannibaL at Hannover Medical School (MHH, Hannover, Germany), communication skills in taking case histories and disclosing diagnoses (breaking bad news) are assessed through an objective structured clinical examination (OSCE). This is part of the examinations which at the $\mathrm{MHH}$ represent the equivalent to the First Part of the Medical Examinations. The second year doctor-patient communication course preparing for these examinations was evaluated during the 2009/10 academic year.

Using questionnaires specific to the learning objectives, learning needs were assessed, pre-post comparisons of self-assessed competencies were performed and key teaching methods were evaluated (5-point Likert scales, " 5 " =fully agree). At T0 (start of the course) 267 students participated (response rate: $93.7 \%$ ), of which 180 filled out the T1 questionnaire during the last session of the course (67.4\%). Withinsubject analyses of variance and paired t-tests were conducted.

The highest learning needs were found for the "to show how"-items regarding history taking and disclosing diagnoses $(M=4.4)$. The T1-TO comparisons showed the greatest improvements for history taking ("to know how": mean difference $=+1.7$, "to show how": $+1.8, p<.0001$ as with all tests) and the "to know how"-item regarding the disclosure of diagnoses (+1.6), followed by the "to show how"-items on disclosing a diagnosis $(+1.4)$, shared decision making $(+1.2)$, self-assessing one's own strengths/weaknesses $(+1.0)$ and confidently approaching new patients $(+0.7)$. Students with T0 values of 1 or 2 on the respective scales improved on average by 2.2 points across all items, students with the value of 3 by 1.1, and from 4 or 5 by 0.1 . Methodically, the use of simulated patients was rated the most helpful $(M=4.8,87 \%$ with the scale value 5).

This doctor-patient communication course is associated with substantial improvements regarding all key learning objectives. Regarding methods, the deployed simulated patients (2-4 per 10-student-course group in 3 of the 7 course sessions, respectively) were rated the most helpful. The present evaluation calls for both further development of the doctor-patient communication curriculum at the $\mathrm{MHH}$ and joint activities across medical schools, which are discussed at the end of the paper.
\end{abstract}

Keywords: Doctor-Patient Communication, Undergraduate Medical Education, Medical History Taking, Diagnosis Disclosure, Health Communication

\section{Introduction}

Both the new version of the German Medical Licensure Act [1], which specifies the teaching of practical experiences in dealing with patients as a new objective in $\S 1$, and the National Competence-Based Learning Objectives for Undergraduate Medical Education (NKLM) [2] have

\author{
Thomas von Lengerke ${ }^{1}$ \\ Angelika Kursch ${ }^{1}$ \\ Karin Lange ${ }^{1}$
}

APG-Teaching

Team $\mathrm{MHH}^{*^{1}}$

1 Hannover Medical School, Medical Psychology Unit, Hannover, Germany 
HannibaL also includes a 28-hour course on doctor-patient communication skills. It is offered by the Medical Psychology Unit and the Medical Sociology Unit in cooperation with the Institute of General Practice, the Clinic for Psychosomatics and Psychotherapy and the Clinical Psychology Working Group of the Clinic of Psychiatry, Social Psychiatry and Psychotherapy. Its learning objectives consist of basic skills in taking patient histories, disclosing diagnoses (breaking bad news), and the use of shared decision-making (SDM) in these contexts. These skills are assessed at two of six stations of an objective structured clinical examination (OSCE). This is part of MHH's internal examinations, which in HannibaL represent the equivalent to the mid-course First Part of the Medical Examinations. The other four OSCE stations test examination techniques which are taught in other parts of the module "Diagnostic Methods" **, to which the communication course belongs. At the same time, in 2010 simulated patients (SP) were used for the third time not only in the OSCE but also in the course itself. Beyond the central evaluation of the total module, it was therefore important to specifically evaluate the course regarding its learning objectives.

\section{Course Concept and Content}

The course includes seven sessions lasting four hours each. The students of each year are divided into 28 groups of ten to allow repeated role play within the group and with the SP. Since its first appearance in the literature [3], the use of SP has become the international standard for the practical study of communication skills in medical studies [4], [5] and is now also popular in Germany [5], [6], [7]. Feedback from the SP is seen an important part of the learning process [4], [8]. The SP used here were primarily recruited amongst members of the German Diabetes Association (Hanover Region), and amongst cardiac patient groups and instructed patients of the Institute of General Practice. A smaller number were drawn from a larger pool of amateur actors which was first set up in 2007 through the mediation of the Amateur Theatre Association of Lower Saxony (in the OSCE amateur actors form the majority of the SP, with each SP used only either in the course or the OSCE). In total, about 80 SP aged 23-77 (61.3\% women) are currently available. They are paid adequately for their services.

Table 1 gives an overview over the seven course sessions. In the first two sessions the basics of doctor-patient communication are taught based on the axioms of Watzlawick [9] and the models by Schulz von Thun [10]. Practical exercises (such as role plays) are used to facilitate active listening, nonverbal communication and initiating communication.

In preparation for the third session, students are instructed to take a biopsychosocial case history with an elderly person (self-study exercise). An appraisal of this takes place in the group at the beginning of the third session, which focuses on the structure and conduct of patient histories in terms of content and practice. Usually, an initial contact in a medical practice is defined as the setting for role plays. For the preparation and appraisal of the role plays, the students use an observation sheet developed by the APG-Teaching Team, which combines the key criteria of the Calgary-Cambridge Guides [11], [4], [12] and FüeßI and Middeke [13]. In the fourth unit two SP are invited for each group of ten, which, through rotation and by splitting of groups, allows for up to eight role plays per course group. To train biopsychosocial history taking, the SP either describe own disease experiences (excluding currently existing symptoms and emergency situations) or use predefined cases.

The focus of the fifth session is the disclosure of diagnoses in a comprehensible and patient-oriented manner based on the Calgary-Cambridge Guides [11], [4], [12] and SPIKES [14]. Through role play students practice breaking bad news as a medical task. Again, a specific observation sheet is used, and - in the sixth session SP facilitate practising. The role plays are based on predefined cases, with their contents adjusted to the SP. They either relate to diabetes mellitus type 2, hypertension, coronary heart disease, disc herniation, chronic bronchitis/COPD, benign prostatic hyperplasia, or breast cancer. These diseases (which relate to a preparatory course at the beginning of HannibaL and other modules) constitute possible themes for the OSCE-station on diagnosis disclosure. In addition to a short patient history, diagnostic findings and the diagnosis itself they contain selected treatment options which offer students the opportunity to practice SDM [15]. Of course, based on a self-administered profile sheet and briefings before ach course sessions the SP have a say on disease patterns they are able and willing to act out. The final session again involves SP and provides opportunities for repetition and exam preparation. Here, the SP are prepared to simulate both contexts (history having and diagnosis disclosure). The course is accompanied by a biweekly lecture (eight sessions of one hour each) to deepen theoretical backgrounds (relating to [16] besides the aforementioned references) and to teach clinical knowledge, partly through patient presentations. Video-assisted learning is part of the course, both through the use of instructional videos for analysis and discussion as well as for recording and appraisal of the students' practical exercises. Written material is usually provided to the students via MHH's elearning platform ILIAS.

This article reports the internal evaluation results of the course during the 2009/10 academic year. Relating to its key learning objectives, it focuses on the learning needs of the students and their competencies at the beginning of the course (TO) as well as their learning progress by the end of the course (T1). The central question is whether the students rate their competencies at T1 as higher than at TO. In addition, key methods such as the use of SP are evaluated. All data are based on self-reports. 
Table 1: Overview of course content and methods (academic year 2009/10)

\begin{tabular}{|c|c|c|}
\hline & Content & Methods* \\
\hline 1. Session & $\begin{array}{l}\text { Basics of doctor-patient } \\
\text { communication } 1 \\
\text { Structure and function of medical } \\
\text { communication: history taking and } \\
\text { diagnosis disclosure (initial contacts) }\end{array}$ & $\begin{array}{l}\text { Introductory presentation (lecturer) } \\
\text { Practical exercises in small groups } \\
\text { (video-assisted) }\end{array}$ \\
\hline 2. Session & $\begin{array}{l}\text { Basics of doctor-patient } \\
\text { communication } 2 \\
\text { Axioms and models of communication } \\
\text { Verbal and nonverbal communication } \\
\text { Basic skills: active listening, introducing } \\
\text { and building trust in doctor-patient } \\
\text { relationships } \\
\text { Multidimensionality of verbal actions }\end{array}$ & $\begin{array}{l}\text { Short introductory presentations on } \\
\text { theory (lecturer) } \\
\text { Practical exercises in small groups } \\
\text { (video-assisted) } \\
\text { Role-plays amongst students }\end{array}$ \\
\hline 3. Session & $\begin{array}{l}\text { Taking case histories } 1 \\
\text { Biopsychosocial history taking following } \\
\text { Calgary-Cambridge Guides [4], [11], } \\
\text { [12] and FüeßI \& Middecke [13] }\end{array}$ & $\begin{array}{l}\text { Evaluation of the self-study task on } \\
\text { taking an older person's patient history } \\
\text { Short introductory lectures (lecturer) } \\
\text { Practical exercises (role-plays) in small } \\
\text { groups }\end{array}$ \\
\hline 4. Session & $\begin{array}{l}\text { Taking case histories } 2 \\
\text { Biopsychosocial history taking following } \\
\text { Calgary-Cambridge Guides [4], [11], } \\
\text { [12] and Füeß| \& Middecke [13] }\end{array}$ & $\begin{array}{l}\text { Role-plays with simulated patients } \\
\text { (simulation of initial contacts) }\end{array}$ \\
\hline 5. Session & $\begin{array}{l}\text { Disclosing diagnoses } 1 \\
\text { Disclosing diagnoses (breaking bad } \\
\text { news) following Calgary-Cambridge } \\
\text { Guides [4], [11], [12]and SPIKES [14] }\end{array}$ & $\begin{array}{l}\text { Short introductory lectures on theory } \\
\text { (lecturer) } \\
\text { Practical exercises (role-plays) in small } \\
\text { groups }\end{array}$ \\
\hline 6. Session & $\begin{array}{l}\text { Disclosing diagnoses } 1 \\
\text { Disclosing diagnoses (breaking bad } \\
\text { news) following Calgary-Cambridge } \\
\text { Guides [4], [11], [12] and SPIKES [14] }\end{array}$ & $\begin{array}{l}\text { Role-plays with simulated patients } \\
\text { (simulation of initial contacts) }\end{array}$ \\
\hline 7. Session & $\begin{array}{l}\text { Repetition ("exam training") } \\
\text { Taking histories and disclosing } \\
\text { diagnoses (breaking bad news) }\end{array}$ & $\begin{array}{l}\text { Role-plays with simulated patients } \\
\text { (simulation of initial contacts) }\end{array}$ \\
\hline
\end{tabular}

\section{Methods}

\section{Questionnaires}

The questionnaires were specifically developed for the learning objectives and oriented towards the competencies tested at the two OSCE stations (history taking and diagnosis disclosure). The T0-questionnaire includes seven items for self-assessment: In accordance with [17] items on knowledge ("to know how") and confidence in one's own abilities ("to show how") regarding history taking and diagnosis disclosure, one "to show how"-item each on self-confidence regarding patient contact and consulting treatment options (SDM), and one regarding self-awareness of one's own strengths and weaknesses in communication (see Table 2). The same sheet also contains a column on the respective learning needs. Finally, it contains a free text field to describe existing experience with patients in the medical field (over and above the nursing internship). Data on age and gender are also collected through the questionnaire.

The second questionnaire (T1) takes up the seven competency items for the pre-post comparison and also includes items to evaluate the use of the SP, video support and quantity of theory and practice. For all items, a 5point Likert scale ranging from 1 (do not agree at all) to
5 (fully agree) was used. In addition, the T1-questionnaire provided students with a free text field giving them the opportunity to list, from their point of view, both positive aspects and suggestions for improvement.

\section{Sample and procedure}

In total 285 students participated in the course in the 2009/10 academic year. The T0-questionnaire was issued at the first course session and completed by 267 students (response rate: $93.7 \%$ ). Of these, 180 students completed the T1 questionnaire at the end of the course (equivalent to $63.2 \%$ of the course participants and $67.4 \%$ of the T0-sample). The survey was conducted anonymously using personal codes. Reasons for non-response at TO are assumed to be organisational in nature, for example due to people changing to the $\mathrm{MHH}$ from other universities at beginning of the summer term (i.e. between the second and third course session) which were admitted to the course. Regarding T1-nonresponse it should be noted that one missed session was permitted per student and that due to the density of examinations towards the end of the summer term, some of the students used the final session as "their" missed session (see the dropout analysis below regarding possible attrition biases). 
Table 2: Questionnaires (left: T0, right: T1)

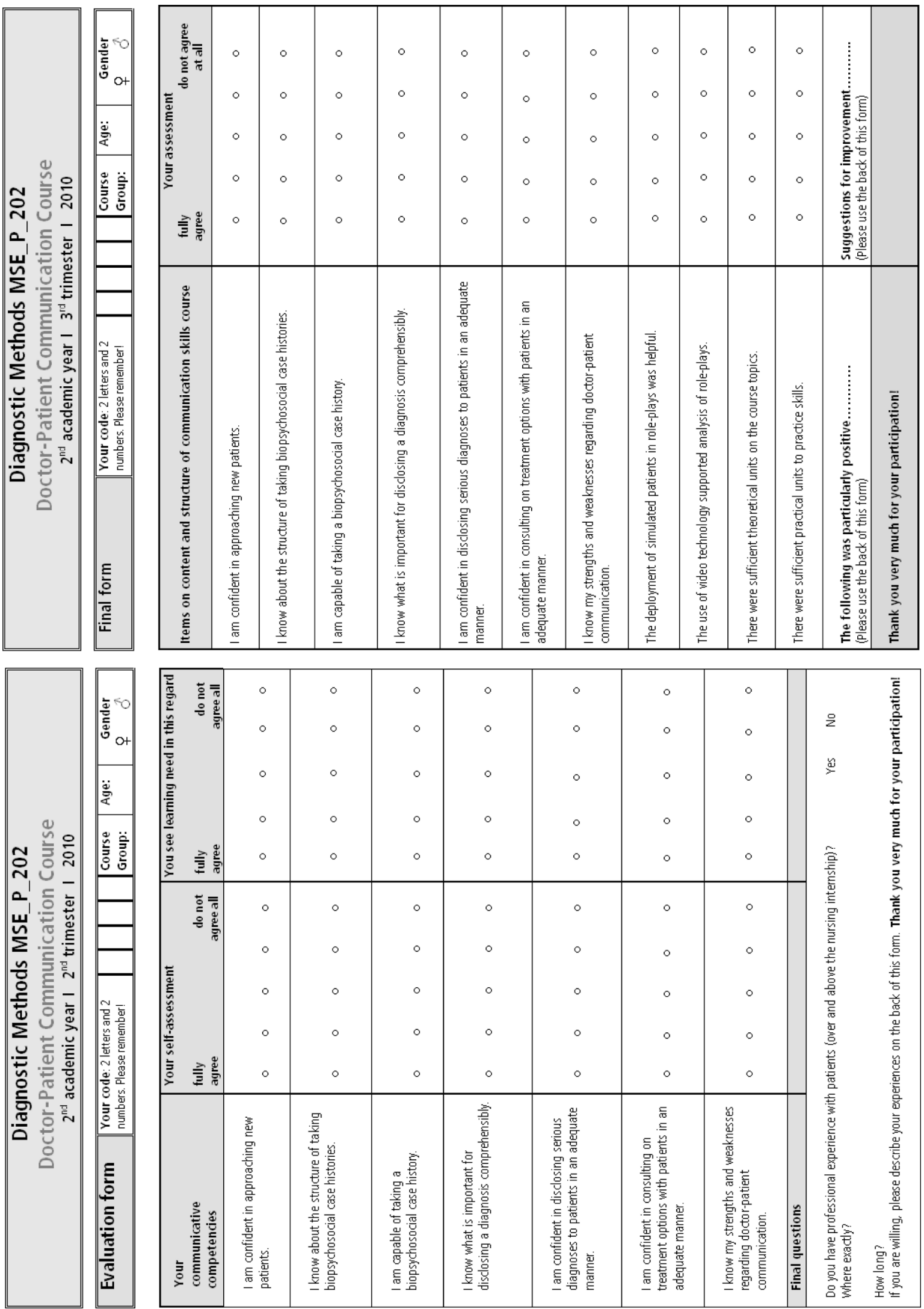

\section{Data analysis}

Following a description of the sample by gender, age and experience with patients, and a T1-dropout analysis, dif- ferences in the learning needs at TO, the competency assessments at T0 and the evaluation of methods at T1 were tested using within-subject analyses of variance. The seven or four items, respectively, defined the repeated measure factor. In all three analyses, the spheri- 
city assumption was tested using Mauchly's sphericity test. This test was always significant $(p<.0001)$ and thus the assumption violated (i.e. in each case unequal variances of the differences of all measurement pairs were shown). As epsilon was $>.75$ in all analyses (learning needs: .87; competency assessments: .91; evaluation of methods: .89), the Huynh-Feldt correction was applied, and the corresponding degrees of freedom and $p$-values are reported. However, divergent conclusions arose neither from the comparison to the model under the assumption of sphericity nor to the multivariate approach. The learning progress regarding each competency (prepost analysis from T0 to T1) was tested using t-tests for paired samples (all analyses: SPSS/PASW Statistics Version 18).

\section{Results}

\section{Sample description and dropout analysis}

Table 3 describes the T0-sample by gender, age and experience with patients. More than two thirds of students are women (68.8\%). They have a larger age variability than male students (standard deviation $=3.6$, vs. 2.4), while on average they do not differ significantly. In total nearly half of the students have experience in dealing with patients over and beyond the nursing internship (49.8\%, with a higher proportion among men than women - $56.4 \%$ vs. $45.7 \%$-, without this difference being significant).

The longitudinal dropout analysis rendered the following results. Of those students with data available at both points in time, $67 \%$ were women, with this proportion being higher in the group with no T1-data (72.7\%), although this was not statistically significant $\left(\mathrm{Chi}^{2}=0.81\right.$, $p=.368$ ). The age difference between the two groups was also not significant $\left(\mathrm{M}=22.8\right.$ vs. $22.9, \mathrm{~F}_{(1.257)}=0.02$, $p=.899)$, as was experience with patients $(46.9 \%$ vs. $55.8 \%, \mathrm{Chi}^{2}=1.85, \mathrm{p}=.174$ ). Regarding learning needs, a significant difference emerged in terms of a higher value in the group with data at T0 and T1 regarding one's own strengths and weaknesses in communication $(M=4.1$ vs. 3.8, $\left.F_{(1.260)}=3.99, p=.047\right)$. The self-assessments of the students' own skills at TO showed differences in the confidence in the ability to take a patient's history $(\mathrm{M}=2.5$ in the group with data for T0 and T1 vs. 2.2, $\mathrm{F}_{(1.264)}=6.19$, $p=.013)$, in knowing how disclose a diagnosis in a comprehensible fashion (3.1 vs. 2.7, $F_{(1,266)}=11: 43, p=.001$ ), and in the confidence in regard to actually disclosing a serious diagnosis (2.7 vs. $2.4, F_{(1.266)}=4.63, p=.032$ ).

\section{Subjective Learning Needs at T0}

As Figure 1 shows, on average students report considerable learning needs with regard to the course's learning objectives. At the same time there were significant differences $\left(\mathrm{F}_{(5.247)} 23.4, \mathrm{p}<.0001\right)$. The highest demand can be found for the "to show how"-items regarding history taking and disclosing diagnoses (both $\mathrm{M}=4.4$ [median=5, not shown here]), followed by the SDM-item (median=5) and the two corresponding knowledge items (median=4). Values for the confidence in approaching new patients and the assessment of the students' own strengths and weaknesses regarding medical communication are at a similar level but slightly lower ( $M=4.0$ and 3.9, median=4).

\section{Self-assessed competencies at T0 and $\mathrm{T} 1$ and learning progress (comparison T1-T0)}

As Figure 2 shows, at T0 the lowest average competency self-assessment emerged for the "to show how"-items regarding history taking and diagnosis disclosure $(\mathrm{M}=2.2$ and 2.4 [median=2, not shown here]), followed by the corresponding knowledge items (median=2 and 3, respectively) and the SDM-item (median=3). Results for confidence in approaching new patients and one's own strengths and weaknesses are somewhat higher ( $\mathrm{M}=3.4$ or 3.1, median=3). Thus, the self-assessments of competencies show an inverse pattern compared to the learning needs (see above). The differences are statistically significant $\left(\mathrm{F}_{(5.429)}=48.6, \mathrm{p}<.0001\right)$.

The T1-T0 comparisons show that significant progress was made regarding all seven learning objectives. The biggest differences were found for the item on history taking ("to know how": mean difference=1.7, "to show how": $1.8, p<.0001$ for all tests) and the "to know how"item on disclosure of diagnoses (1.6). These are followed by the self-assessment of the competency to actually break bad news on a diagnosis (1.4), SDM (1.2), knowing one's own strengths and weaknesses (1.0) and confidently approaching new patients (0.7). At the same time an analysis stratified according to baseline levels showed that students who on the respective scales had rated themselves with the values 1 or 2 on average improved by 2.2 points across all items, those who had rated themselves with 3 improved by 1.1 points, and those with 4 or 5 by 0.1 (not shown).

\section{Evaluation of methods (at T1)}

As Figure 3 shows, students rated the use of the SP as most helpful $(M=4.8)$, with $87 \%$ selecting "fully agree". The theoretical and practical parts of the sessions were rated equally, though slightly lower than the SP $(M=4.2$ and 4.0). In comparison, the use of video technology to record role plays was rated positive by fewer students $(M=3.5)$. The differences were statistically significant $\left(F_{(2.673)}=64.9, p<.0001\right)$. All medians of the items (except regarding the SP) were 4 , respectively. 
Table 3: Sample description $(\mathrm{N}=267)$ by gender, age and experience with patients*

\begin{tabular}{|c|c|c|c|c|c|c|c|c|}
\hline & \multicolumn{2}{|c|}{ Total } & \multicolumn{2}{|c|}{$\begin{array}{c}\text { Female } \\
(\mathrm{N}=176 ; 68.8 \%)\end{array}$} & \multicolumn{2}{|c|}{$\begin{array}{c}\text { Male } \\
(\mathrm{N}=80 ; 31.3 \%)\end{array}$} & & \\
\hline & $n$ & $\%$ & $n$ & $\%$ & $n$ & $\%$ & & \\
\hline Age (in years) & & & & & & & $x^{2}$ & $p$ \\
\hline $18-19$ & 4 & 1,6 & 4 & 2,3 & 0 & 0,0 & & \\
\hline $20-21$ & 111 & 43,0 & 78 & 44,6 & 30 & 39,0 & & \\
\hline $22-23$ & 70 & 27,1 & 39 & 22,3 & 29 & 37,7 & & \\
\hline $24-25$ & 21 & 8,1 & 15 & 8,6 & 5 & 6,5 & 10.4 & .109 \\
\hline $26-27$ & 34 & 13,2 & 23 & 13,1 & 11 & 14,3 & & \\
\hline $28-29$ & 9 & 3,5 & 8 & 4,6 & 1 & 1,3 & & \\
\hline$\geq 30$ & 9 & 3,5 & 8 & 4,6 & 1 & 1,3 & & \\
\hline Mean & 22, & & 23 & & 22,6 & & $\begin{array}{r}\boldsymbol{t} \\
1.1 \\
\boldsymbol{F}\end{array}$ & $\begin{array}{r}p \\
.295 \\
p\end{array}$ \\
\hline Standard deviation & 3,2 & & 3,6 & & 2,4 & & 7.1 & .008 \\
\hline Minimum & 19 & & 19 & & 20 & & & \\
\hline Maximum & 42 & & 42 & & 33 & & & \\
\hline
\end{tabular}

\section{Experience with} patients (except for nursing internship) yes

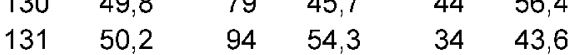

*

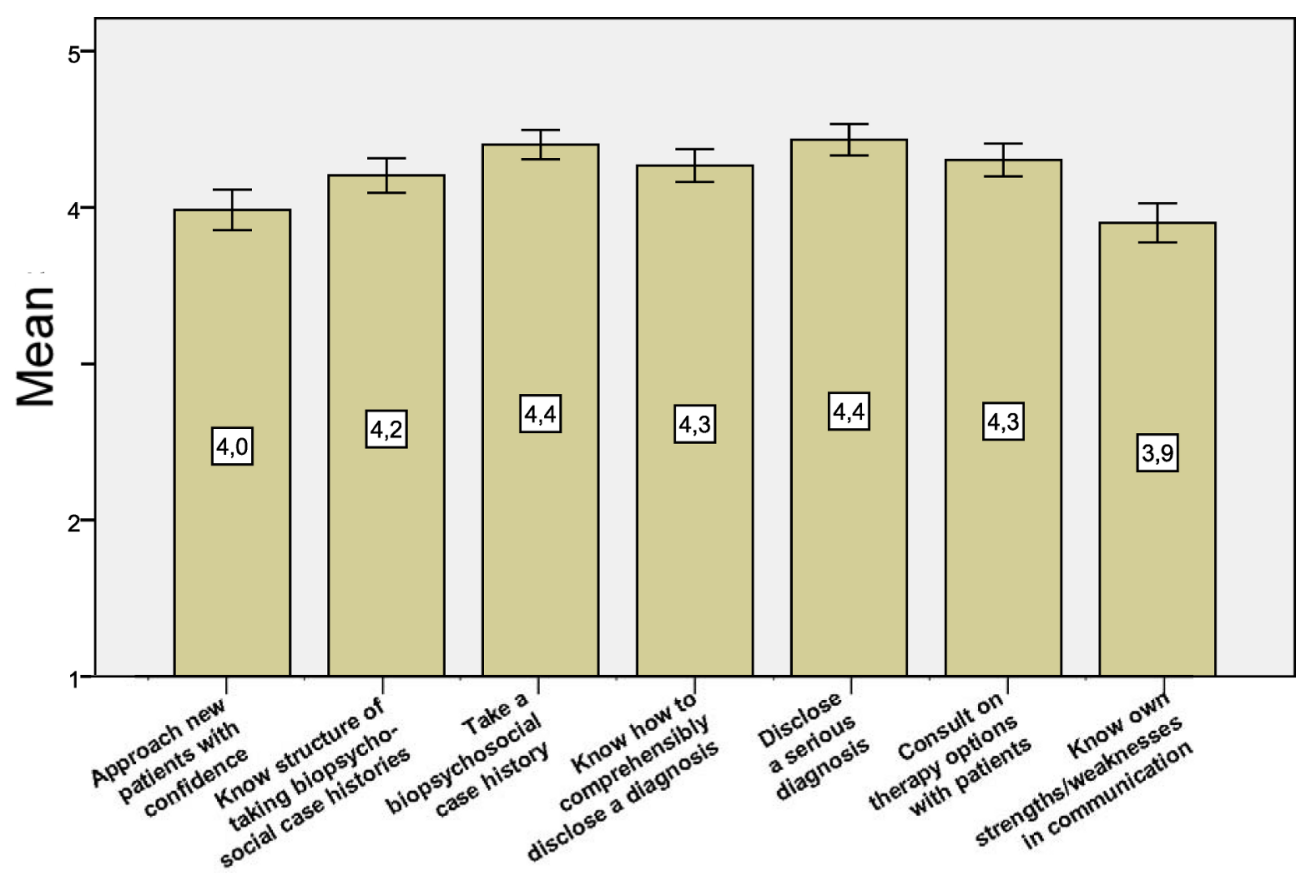

Figure 1: Perceived learning needs at T0

( 1 = fully disagree, 5 = fully agree; mean values with $95 \%$ confidence intervals) 


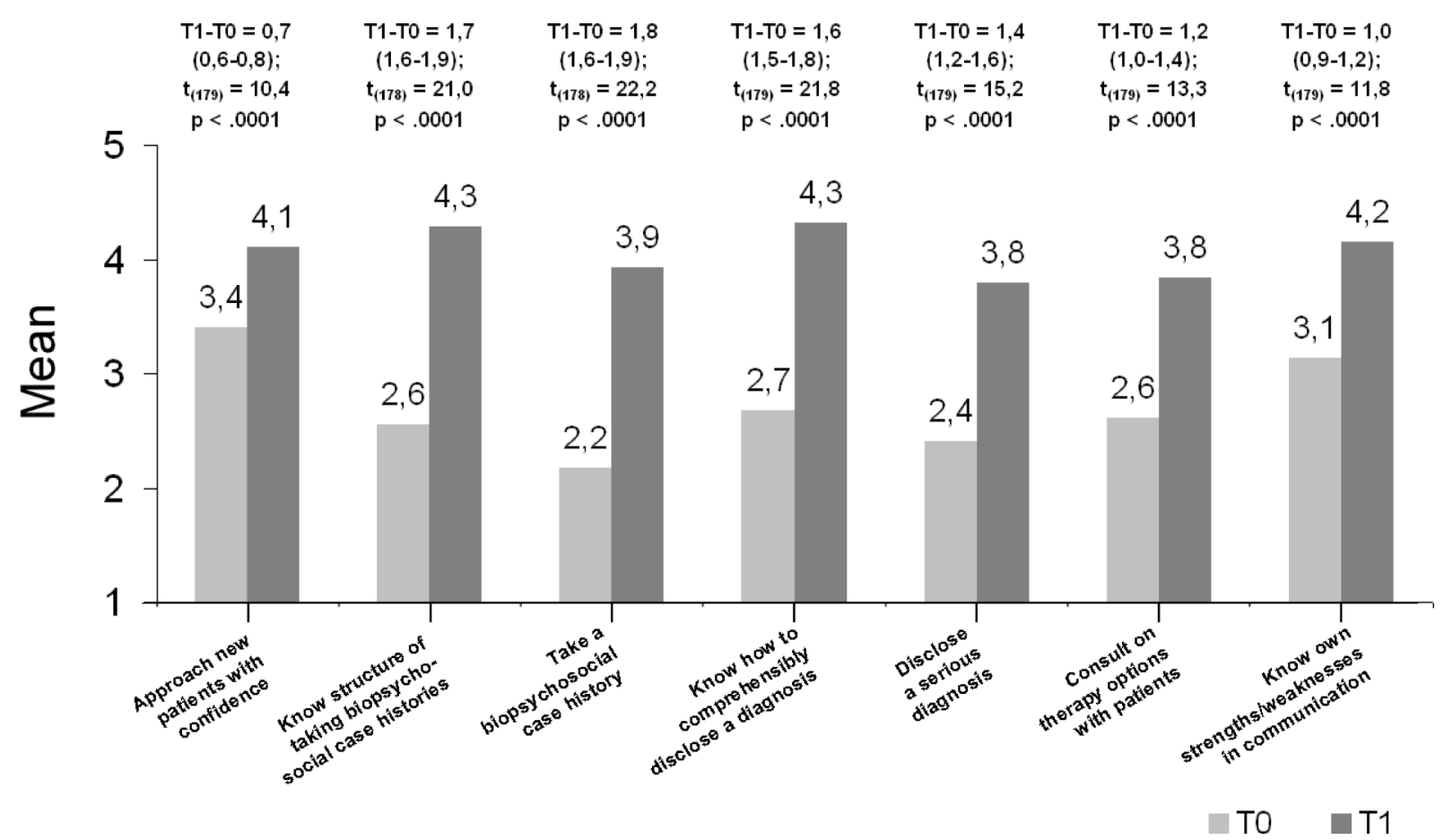

Figure 2: Self-assessments of competencies at T0 and T1 and learning progress (comparison T1-T0)

( 1 = fully disagree, 5 = fully agree; mean differences with $95 \%$ confidence intervals; t-tests for dependent samples, $N=180$, deviating degrees of freedom due to missing values)

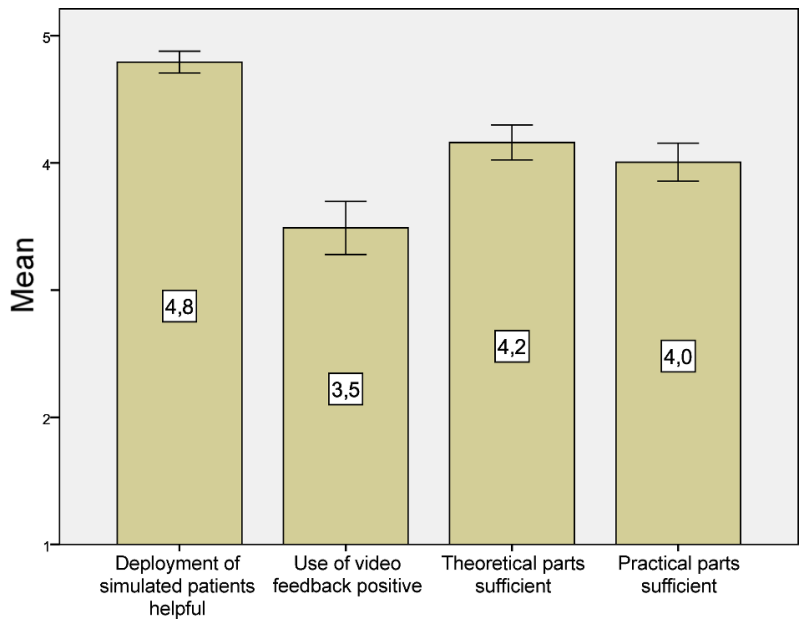

Figure 3: Evaluation of various teaching methods (at T1) ( 1 = fully disagree, 5 = fully agree; mean values with $95 \%$ confidence intervals)

\section{Discussion}

In sum, based on students' self-assessments the doctorpatient communication course in the second year of HannibaL is associated with significant learning progress regarding all its key learning objectives. This is especially true for taking patient histories and disclosing diagnoses (breaking bad news) as its central themes, both in terms of "to know how"- and "to show how"-levels of competency. Taking biopsychosocial case histories showed the strongest learning progress, followed by patient-oriented disclosure of diagnoses, SDM, knowing one's own strengths and weaknesses in communication and the self-confidence in approaching new patients. The latter two had initially shown the lowest learning needs and the best self-assessments. Furthermore, students who had initially rated themselves rather poorly ( 1 or 2 on 5-point scales) on average improved by 2.2 points across all learning objectives, those with intermediate initial selfassessments by 1.1 points, and those with good or optimal T0-values by 0.1 points. Regarding the evaluation of the methods used, the use of SP was rated most positively, followed by the quantity of theory and practice and the use of video feedback.

Before discussing these results in terms of their possible implications for teaching social communication skills in medical school, some particularly methodological limitations of the study should be mentioned. In the first place, it represents a pre-post comparison, and thus only a quasi-experimental design [18]. It was not possible to conduct a (randomised) comparison of the course as the intervention with a control group (e.g. one with "traditional" training in communication skills), which would certainly have been helpful in appraising the results. This, however, is not uncommon in this domain [19], [20], [21], [22], [23], [24], [25], [26], [27], [28], [29] and the pre-post comparison nonetheless allows a judgement of the competence gain. At the same time one might ask which external factors from the students' environment could have improved these specific communication skills. Nevertheless, an earlier study with a control group, which found declines for some parameters in an intervention group with OSCE-training compared to a control group from a previous semester [30], shows that causal interpretations of the course effects in the present study should be treated cautiously. 
Secondly, the mapping of the learning progress is based solely on the students' self-assessments. An objectifying collection of data through performance assessments in the course was not desirable from a didactic point of view, and linking the results to the OSCE-marks was not straightforward for organisational reasons (however, the latter is intended for future academic years by the authors). Strictly speaking, learning objective self-assessments did not operationalise competencies or skills (which would have required observation sheets, SP surveys or theoretical tests [31]) but related self-efficacy expectancies (SEE). SEE are generally defined as the confidence in being able to perform target-oriented behaviour personally [32]. The more self-efficacious a person sees him- or herself the more likely he or she will be capable of performing the behaviour (i.e., showing the skill) in the future. Therefore the above mentioned limitation in this case is at least partly compensated by the fact that strong SEE are also an important intermediary between exercise-induced abilities in medical students and performance in exams such as an OSCE [33]. Thus, it can be assumed that the increased SEE actually have contributed to improved performance, which also could have been shown had pertinent measurements been available (see also [34]).

Thirdly, a self-developed questionnaire was used to model the specific learning objectives of the course as closely as possible. So on the one hand, comparability to other studies is limited and alternatively, it would have been possible to adapt the scales of Parle et al. [35] like, e.g., Ammentorp et al. did [36] (albeit, this would have implied a transfer from continuing training to undergraduate training). On the other hand, further analyses and experience with the present scales will have to show whether the numerically rather low learning progresses amongst students with high competency self-assessments at TO represent ceiling effects or whether modified response scales might be able to show more significant changes in this subgroup as well.

Finally, no T1-questionnaire was received from $32.6 \%$ of the TO-participants. Here, as mentioned before it should be kept in mind that one missed session was permitted and due to the exam density at the end of the summer term, some students chose the final session as their missed session. It could be assumed that participation in T1 was selective in the sense that particularly students with positive self-assessments would have chosen to miss out on the final training session. However, the dropout analysis suggests that this effect was rather weak. Although all three significant (of seven total) comparisons in T0-competencies between T1-participants and nonparticipants showed higher values in the T1-participants, the level of these differences ( 0.3 to 0.4 scale points) is not likely an indication for strong "expert knowledge" on the side of the T1-participants. At the same time, even amongst the T1-participants those with relatively good self-assessments showed learning process, so that given all T0- participants had participated in T1 - overall gains on average would at least not have reduced by negative changes. Since simultaneously gender, age and experience were not significantly associated with dropout, it does not seem to have seriously affected the study's internal validity. Nonetheless, in the future organisational measures will be desirable in order to increase participation rates in $\mathrm{T} 1$, for example by providing an online version of the T1-questionnaire.

Overall, the results of the study indicate that the course contributes significantly to the teaching of social-communicative skills to $\mathrm{MHH}$ students. It takes up learning needs stated by the students. The learning progress as represented in the self-assessments points towards both the appropriateness of the content and methods of the course for the students and their acceptance of these. This is, not least of all, relevant in regards to the placement of the course in the second academic year, as some of the students in the introductory plenary sessions of the course expressed concern regarding insufficient medical knowledge for taking case histories and - in particular disclosing diagnoses. Since this concern was not mentioned in the free text fields of the final questionnaire, it is likely that the course's focus on communication skills was sufficiently successful. Although the OSCE as part of the final module exams certainly will also have had a motivating effect, from our point of view it was possible to impart the necessary orientation to the overall concept and its objectives to the students through the methodical and didactic structures of the course, which allowed consecutive and standardised teaching and learning. The particularly positive evaluation of the SP as a didactic and methodical "medium" is consistent with the results of numerous other studies (for a summary see [4], [6], [7]) and with data from the T1 free text fields which were used by $53 \%$ of students and where 44 of 139 positive responses related to the SP. This encourages consolidation and improvement of this infrastructure, which has been used in preparing students for the OSCE since 2008, e.g. through further training in the feedback skills of the SP [37] and further development of role standards and other quality assurance measures [38]. The absence of criticism in the free text field of the T1-questionnaire regarding the interactive nature of role playing, which includes high levels of experiential learning and performing in front of fellow students [39], also speaks for the course concept. By splitting course groups into small groups in the sessions including SP as well and the explicit training of constructive feedback, students were introduced to performing in front of a group right from the outset of the course.

The significantly poorer (though still positive) evaluation of the amounts of theoretical and practical parts in the sessions are also reflected in the free text field data where from a total of 73 proposals for improvement 43 mention the need for more practice and less theory. Perhaps this balance is not optimal to date, especially with regard to the learning goals of communicating (among others) serious diagnoses, and as this is only specifically addressed as a topic from the fifth course session onwards. This has already led conceptual changes 
as part of the quality assurance and continuous development of the course concept following the 2009/10 academic year. These changes aim for the consistent teaching of the basics of medical communication using the topics of taking medical histories ( $1^{\text {st }}$ course session) and breaking bad news ( $2^{\text {nd }}$ session) so as to focus on the topics right from the outset.

Finally the rather moderately positive assessment of the use of video feedback points to another need for improvement in the course concept. Although this method has proven valuable in teaching communication skills [4], [24], it significantly increases the amount of time needed due to the presentation and discussion of the recordings. In this context, it is our experience that students tend to favour increasing the amount of role play against video analysis. It is a task for the development of the course concept to integrate this method more consistently and achieve a better balance between in-depth analysis and the number of simulations. In addition, infrastructure such as observation rooms with appropriate equipment (and mirrored glass walls such as in the Study Hospital Munster ${ }^{\circledR}$ ) would be beneficial.

A further evaluation of the free text fields pointed towards "atmospheric" reasons for the success of the course. The professional, educational and personal competence of the tutors was positively mentioned in 51 cases. 14 mentions described the group size of 10 students as optimal. In addition, the opportunity for experiential learning in a way that respects individuality and the low threshold for more introverted students to get involved were perceived positively. More partner interviews and small group work within the groups of 10 were called for in nine cases.

Analyses stratified by gender and experience were not reported because they revealed only marginal differences. For example, the pre-post comparisons only showed a gender difference regarding confidence in approaching new patients, with men at TO having slightly higher selfassessments ( $M=3.8$ vs. 3.2) and women reporting slightly higher significant learning progress (0.8 vs. 0.5 ; $p=.032$ ). All other tests were not significant, as with the evaluation of methods. The mean differences of all learning needs, with a maximum of 0.4 points, were lower than the learning progress. All told, the differences indicate slightly more positive self-assessments of male students, which is consistent with more pronounced medical ideals in female medical students [40]. Nonetheless, gender-specific aspects in the present cohort of students appear of marginal relevance regarding the acquisition of communicative skills.

Overall, the positive evaluation of the communication course in our view represents a further empirical argument for a longitudinal social communication and doctorpatient communication curriculum at the $\mathrm{MHH}$, which integrates and further develops existing curricula in a learning spiral [41]. In the long term, this could include evaluations which study the effects of such a curriculum on the subsequent performance of students and postgraduate doctors during the Practical Year or in profes- sional practice (particularly as such effects are not without preconditions [42]).

In addition, we agree with the recommendations to strengthen cross-university and cross-faculty activities, not least of all in terms of valid and reliable examination methods [43]. This is supported by the expected efficiency gains. These are not least of all important because the implementation of teaching concepts such as the one evaluated here (practice-oriented teaching and learning in small groups of students and using SP) requires significant personnel, organisational and financial resources. It is plausible that such efforts are particularly sustainable if longitudinal curricula covering all academic years become the standard in this area of competence. Ideally such (interdisciplinary is possible) activities are based on a consensus framework. A blueprint for such a model could be the British consensus paper [44]. It begins by defining respect as the basis of effective clinical communication and by discussing theory and evidence, the role of clinical communication, specific topics such as behaviour modification, media and communication beyond the patient lists virtually all relevant key issues. We hope that this evaluation study of one curricular block at the $\mathrm{MHH}$ will add to this overall process.

\section{Notes}

*APG-Teaching Team MHH (Doctor-Patient Communication Teaching Team MHH): Karin Lange (Coordinator), Ulrich Brinkmeier, Gundula Ernst, Angelika Kursch, Thomas von Lengerke, Thomas Schneller (Medical Psychology Unit); Siegfried Geyer (Coordinator), Anja Löbel, Friederike Otto, Stefanie Sperlich (Medical Sociology Unit); Eva Hummers-Pradier, Carsten Kruschinski, Heidrun Lingner, Christiane Müller, Erika Penner (Institute for General Practice); Uwe Hartmann, Susanne Philippsohn, Lotta Winter (Clinical Psychology Working Group at the Clinic for Psychiatry, Social Psychiatry and Psychotherapy); Burkard Jäger, Maike Möllenkamp, Angela Trieschmann (Clinic for Psychosomatics and Psychotherapy)

**Coordinator of the Longitudinal Internal Medicine Curriculum : Prof. Dr. Hermann Haller, M.D. (who has also lead the development of HannibaL as MHH's Dean of Medicine and Postgraduate and Complementary Studies); Coordinator of the Module „Diagnostic Methods“: Dr. Saskia Merkel, M.D..

\section{Acknowledgement}

We would like to thank the students of the model medical curriculum HannibaL who started studying in 2008 for their participation in the study, and thus their willingness to complete yet another questionnaire in addition to the surveys conducted by the Evaluation Office at Hannover Medical School's Academic Dean's Office. 


\section{Competing interests}

The authors declare that they have no competing interests.

\section{References}

1. Bundesministerium der Justiz. Approbationsordnung für Ärzte vom 27. Juni 2002 (BGBI. I S. 2405), die zuletzt durch Artikel 10 des Gesetzes vom 24. Juli 2010 (BGBI. I S. 983) geändert worden ist. Berlin: Bundesministerium der Justiz; 2010. Zugänglich unter/available from: http://www.gesetze-iminternet.de/_appro_2002/BJNR240500002.html

2. Hahn EG, Fischer MR. Nationaler Kompetenzbasierter Lernzielkatalog Medizin (NKLM) für Deutschland: Zusammenarbeit der Gesellschaft für Medizinische Ausbildung (GMA) und des Medizinischen Fakultätentages (MFT). GMS Z Med Ausbild. 2009;26(3):Doc35. DOI: 10.3205/zma000627

3. Barrows HS. Simulated patients in medical training. Can Med Assoc J. 1968;98(14):674-676.

4. Kurtz S, Silverman J, Draper J. Teaching and learning communicating skills in medicine. 2nd edition. Oxford: Radcliffe; 2004.

5. Fröhmel A, Burger W, Ortwein H. Einbindung von Simulationspatienten in das Studium der Humanmedizin in Deutschland. Dtsch Med Wochenschr. 2007;132(11):549-554. http://dx.doi.org/10.1055/s-2007-970375

6. Rockenbauch K, Fabry G, Petersen C, Daig I, Philipp S. Der Einsatz von Schauspielpatienten in der Medizinischen Psychologie - allgemeiner Überblick und konkrete Umsetzungsbeispiele. Z Med Psychol. 2008;17(4):185-192.

7. Simmenroth-Nayda A, Chenot JF, Fischer Th, Scherer M, Stanske $\mathrm{B}$, Kochen MM. Mit Laienschauspielern das ärztliche Gespräch trainieren. Dtsch Arztebl. 2007;104(13):A847-852.

8. Bokken L, Rethans J-J, Jöbsis O, Duvivier R, Scherpbier A, van der Vleuten $C$. Instructiveness of real patients and simulated patients in undergraduate medical education: a randomized experiment. Acad Med. 2010;85(1):148-154. http://dx.doi.org/ 10.1097/ACM.0b013e3181c48130

9. Watzlawick P, Beavin JH, Jackson DD. Menschliche Kommunikation: Formen, Störungen, Paradoxien. Bern: Huber; 1969.

10. Schulz von Thun F. Miteinander reden. Reinbek: Rowohlt; 2008.

11. Kurtz S, Silverman J, Benson J, Draper J. Marrying content and process in clinical method teaching: enhancing the CalgaryCambridge Guides. Acad Med. 2003;78(8):802-809. http:// dx.doi.org/10.1097/00001888-200308000-00011

12. Silverman J, Kurtz S, Draper J. Skills for communicating with patients. 2nd edition. Oxford: Radcliffe; 2005.

13. FüeßI HS, Middeke M. Anamnese und klinische Untersuchung. 4. Auflage. Stuttgart: Thieme; 2010.

14. Baile W, Buckman R, Lenzi R, Glober G, Beale EA, Kudelka AP. SPIKES-A six-step protocol for delivering bad news: application to the patient with cancer. Oncologist. 2000;5(4):302-311. http://dx.doi.org/10.1634/theoncologist.5-4-302

15. Scheibler $F$, Janssen $C$, Pfaff $H$. Shared decision making: ein Überblicksartikel über die internationale Forschungsliteratur. Soz Praventivmed. 2003;48(1):11-23. http://dx.doi.org/ $10.1007 / \mathrm{s} 000380300002$

16. Buser K, Schneller T, Wildgrube K. KLB Medizinische Psychologie - Medizinische Soziologie. 6. Auflage. München: Elsevier/Urban \& Fischer; 2007.
17. Miller GE. The assessment of clinical skills/competence/performance. Acad Med. 1990;65(9 Suppl):S63-67. http://dx.doi.org/10.1097/00001888199009000-00045

18. Mohr LB. Impact analysis for program evaluation. Chicago, II: Dorsey Press; 1988.

19. Bahrs O. Kommunikationsförderung in der Vor-Klinik - Kursus der Medizinischen Psychologie und Medizinischen Soziologie. Z Med Psychol. 2010;19(2):97-101.

20. Brenk-Franz K, Kubieziel H, Schulz S, Gensichen J. Simulationspatienten in der allgemeinmedizinischen Lehre: Das Jenaer Programm - eine Pilotstudie. Z Allg Med. 2010;86(4):152158.

21. Fischbeck S, Mauch M, Leschnik E, Laubach W. Entwicklung und Evaluation einer OSCE für die Überprüfung kommunikativer ärztlicher Kompetenz im Kursus der Medizinischen Psychologie und Medizinischen Soziologie. Z Med Psychol. 2010;19(2):9496.

22. Fischer T, Chenot J-F, Kleiber C, Kochen MM, Simmenroth-Nayda A, Staats H, Hermann-Lingen C. Kurs „ärztliche Basisfähigkeiten“ - Evaluation eines primärärztlichen orientierten Unterrichtskonzeptes im Rahmen der neuen Approbationsordnung. GMS Z Med Ausbild. 2005;22(3):Doc59. Zugänglich unter/available from: http://www.egms.de/de/ journals/zma/2005-22/zma000059.shtml

23. Koerfer A, Köhle K, Obliers R, Sonntag B, Thomas W, Albus C. Training und Prüfung kommunikativer Kompetenz. Aus- und Fortbildungskonzepte zur ärztlichen Gesprächsführung. Gesprächsforschung. 2008;9:34-78. Zugänglich unter/available from: http://www.gespraechsforschung-ozs.de/heft2008/agkoerfer.pdf

24. Kopecky-Wenzel M, Maier EM, Muntau AC, Reinhardt D, Frank R. Überbringen schlechter Nachrichten - videogestützte Trainingseinheit für Medizinstudenten. Z Kinder Jugendpsychiatr Psychother. 2009;37(2):139-144. http://dx.doi.org/10.1024/ 1422-4917.37.2.139

25. Mitzkat A, Schulz C, Kasenda B, Langer T, Schnell MW. „ARZT IM GANZEN SPREKTRUM“: Die INTEGRIERTEN CURRICULA der Medizinerausbildung an der Universität Witten/Herdecke Rückblick auf sechs Jahre Lehre im Hinblick auf Praxisorientiertung und theoretische Vorgaben. GMS Z Med Ausbild. 2006;23(4):Doc66. Zugänglich unter/available from: http://www.egms.de/static/de/journals/zma/2006-23/ zma000285.shtml

26. Petersen C, Busche W, Bergelt C, Huse-Kleinstoll G. Kommunikationstraining als Teil des Medizinstudiums: Ein Modellversuch. GMS Z Med Ausbild. 2005;22(1):Doc08. Zugänglich unter/available from: http://www.egms.de/static/ de/journals/zma/2005-22/zma000008.shtml

27. Philipp S, Merten K. Erfahrungen mit Standardisierten Patienten im neuen Lehrkonzept zur Vermittlung kommunikativer Kompetenzen im Fach Medizinische Psychologie - das Jenaer Modell. Z Med Psychol. 2006;15(1):11-19.

28. Pucher-Matzner I, Gleiss A, Schmidts M, Frischenschlager O. Die Checkliste PD „Professionelles ärztliches Kommunikationsverhalten“ in Unterricht und Evaluation kommunikativer Fertigkeiten im Medizinstudium. GMS Z Med Ausbild. 2006;23(4):Doc68. Zugänglich unter/available from: http://www.egms.de/static/de/journals/zma/2006-23/ zma000287.shtml

29. Wiesmann U, Niehörster G, Hannich H-J. Medizinpsychologische Lehre innovativ gestalten: Förderung von Kommunikations- und Forschungskompetenzen in der vorklinischen Ausbildung. Z Med Psychol. 2009;18(2):88-96. 
30. Kopp V, Schewe S. Kann durch Training Anamnese und klinische Untersuchung vermittelt werden? GMS Z Med Ausbild. 2005;22(1):Doc15. Zugänglich unter/available from: http:// www.egms.de/static/de/journals/zma/2005-22/ zma000015.shtml

31. Duffy FD, Gordon GH, Whelan G, Cole-Kelly K, Frankel R; All Participants in the American Academy on Physician and Patient's Conference on Education and Evaluation of Competence in Communication and Interpersonal Skills. Assessing competence in communication and interpersonal skills: the Kalamazoo II Report. Acad Med. 2004;79(6):495-507. http://dx.doi.org/ 10.1097/00001888-200406000-00002

32. Bandura A. Self-efficacy: the exercise of control. New York: Freeman; 1997.

33. Mavis B. Self-efficacy and OSCE performance among second year medical students. Adv Health Sci Educ. 2001;6(2):93-102. http://dx.doi.org/10.1023/A:1011404132508

34. Schultz J-H, Schönemann J, Lauber H, Nikendei C, Herzog W, Jünger J. Einsatz von Simulationspatienten im Kommunikationsund Interaktionstraining für Medizinerinnen und Mediziner (MediKIT): Bedarfsanalyse - Training - Perspektiven. Gruppendynamik Organisationsberatung. 2007;38(1):7-23. http://dx.doi.org/ 10.1007/s11612-007-0002-y

35. Parle M, Maguire P, Heaven C. The development of a training model to improve health professionals' skills, self-efficacy and outcome expectancies when communicating with cancer patients. Soc Sci Med. 1997;44(2):231-240. http://dx.doi.org/10.1016/ S0277-9536(96)00148-7

36. Ammentorp J, Sabroe S, Kofoed P-E, Mainz J. The effect of training in communication skills on medical doctors' and nurses self-efficacy: a randomized controlled trial. Patient Educ Couns. 2007;66(3):270-277. http://dx.doi.org/10.1016/ j.pec.2006.12.012

37. Bokken L, Linssen T, Scherpbier A, van der Vleuten C, Rethans JJ. Feedback by simulated patients in undergraduate medical education: a systematic review of the literature. Med Educ. 2009;43(3):202-210. http://dx.doi.org/10.1111/j.13652923.2008.03268.x

38. Cleland JA, Abe K, Rethans J-J. The use of simulated patients in medical education: AMEE Guide No 42. Med Teach. 2009;31(6):477-486. http://dx.doi.org/10.1080/ 01421590903002821

39. Dieckmann P, Rall M, Eich C, Schnabel K, Jünger J, Nikendei C. Rollenspiel als wesentliches Element bei Simulationsverfahren in der Medizin. Z Evid Fortbild Qual Gesundhwes. 2008;102(10):642-647. http://dx.doi.org/10.1016/ j.zefq.2008.11.020

40. Schrauth M, Kowalski A, Weyrich P, Begenau J, Werner A, Zipfe S, Nikendel C. Selbstbild, Arztbild und Arztideal: Ein Vergleich Medizinstudierender 1981 und 2006. Psychother Psych Med. 2009;59(12):446-453. http://dx.doi.org/10.1055/s-0029 1202343

41. Bachmann C, Hölzer H, Dieterich A, Fabry G, Langewitz W, Lauber $\mathrm{H}$, Ortwein H, Pruskil S, Schubert S, Sennekamp M, SimmenrothNayda A, Silbernagel W, Scheffer S, Kiessling C. Longitudinales, bologna-kompatibles Modell-Curriculum „Kommunikative und soziale Kompetenzen“: Ergebnisse eines interdisziplinären Workshops deutschsprachiger medizinischer Fakultäten. GMS Z Med Ausbild. 2009;26(4):Doc38. DOI: 10.3205/zma000631
42. Stimmel B, Cohen D, Fallar R, Smith L. The use of standardised patients to assess clinical competence: does practice make perfect? Med Educ. 2006;40(5):444-449 http://dx.doi.org/ 10.1111/j.1365-2929.2006.02446.x

43. Kiessling C, Dieterich A, Fabry G, Hölzer H, Langewitz W, Mühlinghaus I, Pruskil S, Scheffer S, Schubert S. Basler Consensus Statement „Kommunikative und soziale Kompetenzen im Medizinstudium“: Ein Positionspapier des GMA-Ausschusses Kommunikative und soziale Kompetenzen. GMS Z Med Ausbild. 2008;25(2):Doc83. Zugänglich unter/available from: http:// www.egms.de/static/de/journals/zma/2008-25/ zma000567.shtm

44. von Fragstein M, Silverman J, Cushing A, Quilligan S, Salisbury $\mathrm{H}$, Wiskin C; UK Council for Clinical Communication Skills Teeaching in Undergraduate Medical Education. UK consensus statement on the content of communication curricula in undergraduate medical education. Med Educ. 2008;42(11):1100-1107. http://dx.doi.org/10.1111/j.13652923.2008.03137.x

\section{Corresponding author:}

PD Dr. phil. Thomas von Lengerke

Hannover Medical School, Medical Psychology Unit (OE 5430), Carl-Neuberg-Straße 1, 30625 Hannover, Germany, Phone: +49 (0)511/532-4445, Fax: +49

(0)511/532-4214

lengerke.thomas@mh-hannover.de

\section{Please cite as}

von Lengerke T, Kursch A, Lange K, APG-Teaching Team MHH*. The communication skills course for second year medical students at Hannover Medical School: An evaluation study based on students' self-assessments. GMS Z Med Ausbild. 2011;28(4):Doc54. DOI: 10.3205/zma000766, URN: urn:nbn:de:0183-zma0007668

\section{This article is freely available from}

http://www.egms.de/en/journals/zma/2011-28/zma000766.shtml

Received: 2011-01-27

Revised: 2011-08-10

Accepted: 2011-09-08

Published: 2011-11-15

\section{Copyright}

(C2011 von Lengerke et al. This is an Open Access article distributed under the terms of the Creative Commons Attribution License ( $h$ ttp://creativecommons.org/licenses/by-nc-nd/3.0/deed.en). You are free: to Share - to copy, distribute and transmit the work, provided the original author and source are credited. 